Propriedades de filtros lineares para sistemas lineares com saltos markovianos a tempo discreto 



\title{
Propriedades de filtros lineares para sistemas lineares com saltos markovianos a tempo discreto
}

\author{
Maria Josiane Ferreira Gomes
}

Orientador: Prof. Dr. Eduardo Fontoura Costa

Tese apresentada ao Instituto de Ciências Matemáticas e de Computação - ICMC-USP, como parte dos requisitos para obtenção do título de Doutor em Ciências - Ciências de Computação e Matemática Computacional. VERSÃO REVISADA. 
Ficha catalográfica elaborada pela Biblioteca Prof. Achille Bassi e Seção Técnica de Informática, ICMC/USP, com os dados fornecidos pelo(a) autor(a)

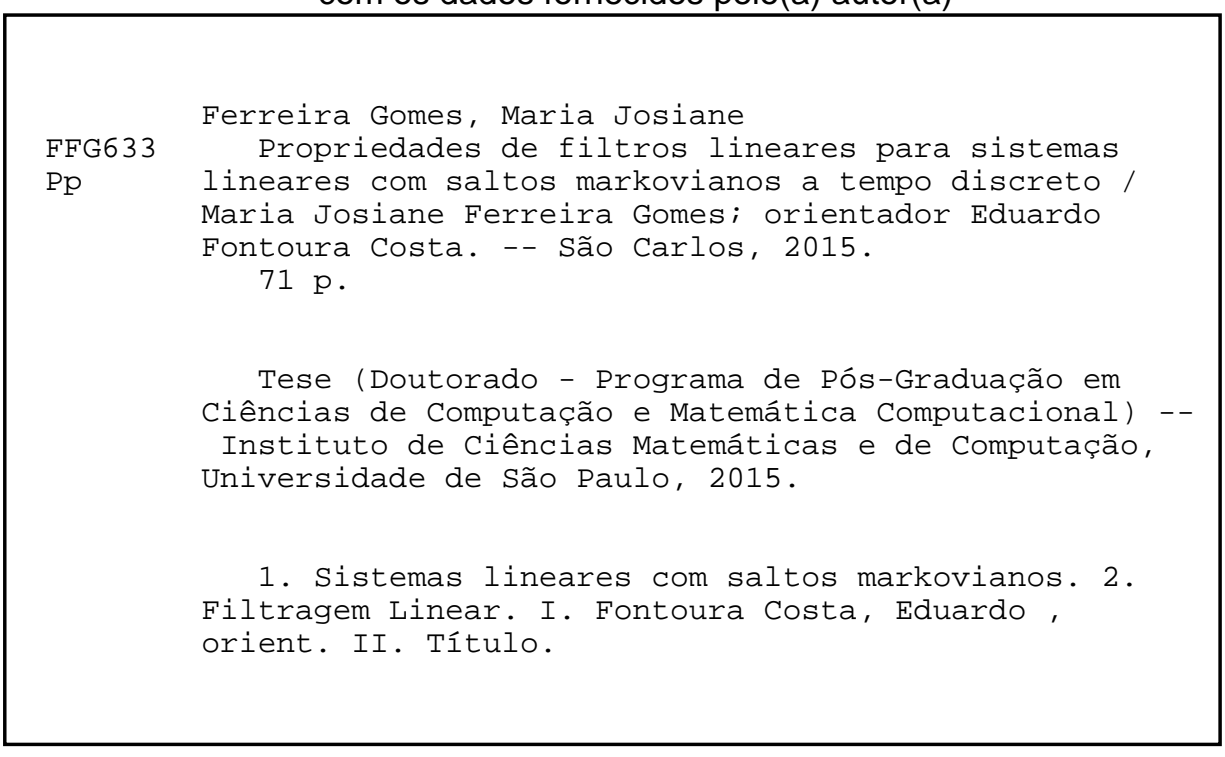


À Gisele A. Bráz de Lima (in memorian)

"Amigo é coisa pra se guardar Debaixo de sete chaves, Dentro do coração ..."

Milton Nascimento. 



\section{Agradecimentos}

Expressarei em poucas linhas minha gratidão àqueles que de alguma forma estiveram presentes e que muito me ajudaram em meu caminho até aqui.

À Deus, pela constante e amorosa presença.

À minha família, que sempre me deu suporte de forma incondicional em todos os aspectos possíveis durante meus estudos.

À Letricia e Luciano, pela lealdade, amizade e atenção.

Ao Prof. Dr. Eduardo Fontoura Costa, pela amizade, competência, seriedade e pela forma generosa com que compartilhou seus conhecimentos nestes anos de trabalho.

Ao CNPq - Conselho Nacional de Desenvolvimento Científico e Tecnológico, pelo suporte financeiro. Processo $n^{\circ} .142174 / 2010-6$.

À FAPESP - Fundação de Amparo à Pesquisa do Estado de São Paulo, pelo apoio financeiro não menos importante. Processo ${ }^{\circ}$. 2013/19380 - 8 .

À todos os professores e funcionários do Instituto de Ciências Matemáticas e de Computação da Universidade de São Paulo.

Meus sinceros agradecimentos. 



\section{Resumo}

Este trabalho é dedicado ao estudo do erro de estimação em filtragem linear para sistemas lineares com parâmentros sujeitos a saltos markovianos a tempo discreto. Indroduzimos o conceito de alcançabilidade média para uma classe de sistemas. Construímos um conjunto de matrizes de alcançabilidade e mostramos que o conceito usual de alcançabilidade definido através da positividade do gramiano é caracterizado pela definição por posto completo destas matrizes. A alcançabilidade média funciona como condição necessária e suficiente para positividade do segundo momento do estado do sistema, resultado esse que auxilia na caracterização da positividade uniforme da matriz de covariância do erro de estimação. Abordamos a estabilidade de estimadores com a interpretação de que a covariância do erro permanece limitada na presença de erro de qualquer magnitude no modelo do ruído, que é uma característica relevante para aplicações. Apresentamos uma prova de que filtros markovianos são estáveis sempre que o segundo momento condicionado é positivo. Exemplos numéricos encontram-se inclusos.

Palavras-chave: Sistemas lineares com saltos markovianos, alcançabilidade média, positividade, filtros lineares, estabilidade de filtros markovianos. 



\section{Abstract}

This work studies linear filtering for discrete-time systems with Markov jump parameters. We introduce a notion of average reachability for these systems and present a set of matrices playing the role of reachability matrices, in the sense that their rank is full if and only if the system is average reachable. Reachability is also a sufficient condition for the second moment of the system to be positive. Uniform positiveness of the error covariance matrix is studied for general (possibly non-markovian) linear estimators, relying on the state second moment positiveness. Stability of linear markovian estimators is also addressed, allowing to show that markovian estimators are stable whenever the system is reachable, with the interpretation that the error covariance remains bounded in the presence of error of any magnitude in the model of the noise, which is a relevant feature for applications. Numerical examples are included.

Keywords: Linear systens with jumping parameters, average reachability, positivity, linear estimators, markovian filters satability. 


\section{Sumário}

1 Introdução 1

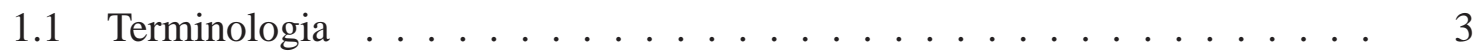

1.2 Abordagem . . . . . . . . . . . . . . . . . . . 4

1.3 Interpretação física e detalhes técnicos . . . . . . . . . . . . . . 5

1.4 Organização do texto . . . . . . . . . . . . . . . . . . 6

2 Notações, definições e resultados preliminares 9

2.1 Notações . . . . . . . . . . . . . . . . . . . . . . . . . . 9

2.1.1 Propriedades usuais do valor esperado . . . . . . . . . . . . . . 10

2.2 Cadeia de Markov . . . . . . . . . . . . . . . . . . . . . . . . . 11

2.2.1 Propriedades da cadeia de Markov . . . . . . . . . . . . . . . . 12

2.3 Sistemas lineares com saltos markovianos . . . . . . . . . . . . . . 13

2.3.1 Segundo momento de $x(k) \ldots \ldots \ldots \ldots$

2.4 Filtros lineares . . . . . . . . . . . . . . . . . . 15

2.5 Filtros lineares markovianos f................. 17

3 M-alcançabilidade e matrizes de M-alcançabilidade 19

3.1 M-alcançabilidade, gramiano e matrizes de M-alcançabilidade . . . . . . . 19

3.1.1 Propriedades do $\mathcal{K} \operatorname{er}\left\{R_{\ell, i}(k)\right\} \ldots \ldots \ldots 22$

3.2 O Teorema do posto completo . . . . . . . . . . . 24

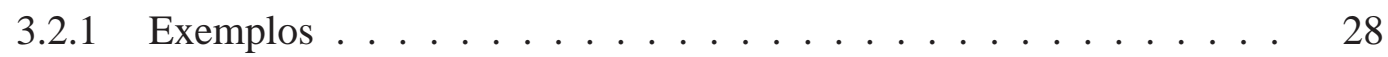

4 Positividade do segundo momento 31

4.1 Resultados auxiliares e ordenação de matrizes . . . . . . . . . . . . . . 31 
4.1.1 Matrizes de M-alcançabilidade e segundo momento . . . . . . . . . 32

4.1 .2 Ordenação de matrizes . . . . . . . . . . . . . . . . . . 32

4.2 Positividade do processo $X(k) \ldots \ldots \ldots \ldots$

5 Positividade do processo de erro do estimador 39

5.1 Positividade do processo $\mathrm{Y}(\mathrm{k}) \ldots \ldots \ldots$

5.2 M-alcançabilidade e cadeias ergódicas . . . . . . . . . . . . . . 46

5.2 .1 Exemplos ilustrativos . . . . . . . . . . . . . . 46

$6 \quad$ Estabilidade de filtros lineares markovianos 49

6.1 Exemplos ilustrativos . . . . . . . . . . . . . . . . . 57

7 Conclusão

Referências Bibliográficas $\quad 66$ 


\section{Lista de Figuras}

2.1 Representação da cadeia de Markov com três estados. . . . . . . . . . . . . 11

5.1 Calculo dos valores $v \mathcal{E}\{X(k)\} v^{\prime}$ (denotada com o) e covariância do erro de estimação $v \mathcal{E}\{Y(k)\} v^{\prime}($ denotada por $\times) \ldots \ldots$. . . . . . . . . 47

5.2 Cálculo dos valores de $v \mathcal{E}\{X(k)\} v^{\prime}$ (denotada com o) e covariância do erro de estimação $v \mathcal{E}\{Y(k)\} v^{\prime}($ denotada por $\times) \ldots \ldots . \ldots . . . \ldots 47$

6.1 Estimação da Covariância de erro de Exemplo 6.1. $\mathcal{E}\left\{Y^{k}(D, E, \Sigma)\right\}$ (denotada com o) e $\mathcal{E}\left\{Y^{k}(D, B, \Psi)\right\}($ detotada com $\times)$. . . . . . . . . . 57

6.2 Estimação da Covariância de erro de Exemplo 6.2. $\mathcal{E}\left\{Y^{k}(D, E, \Sigma)\right\}$ (denotada com $\circ)$ e $\mathcal{E}\left\{Y^{k}(D, B, \Psi)\right\}($ detotada com $\times)$. . . . . . . . . . 58

6.3 Estimação da Covariância de erro de Exemplo 6.2. $\mathcal{E}\left\{Y^{k}(D, E, \Sigma)\right\}$ (denotada com $\circ)$ e $\mathcal{E}\left\{Y^{k}(D, B, \Psi)\right\}($ detotada com $\times)$. . . . . . . . . 58 


\section{Lista de Símbolos}

$\begin{array}{ll}\mathcal{E}(\cdot) & \text { Valor esperado } \\ O(\cdot) & \text { Notação de Landau } \\ E, \Sigma & \text { Valores nominais } \\ B, \Psi & \text { Valores reais } \\ (A, E, P) & \text { SLSM com valores nominais e matriz de transição } P \\ (A, B, P) & \text { SLSM com valores reais e matriz de transição } P \\ X_{t}^{k}(\Sigma, B) & \text { Covariância do estado } x(k) \text { para os valores nominais } E \text { e } \Sigma \text { no intervalo de } \\ & \text { tempo }[t, k] \\ X^{k}(\Sigma, B) & \text { Covariância do estado } x(k) \text { para os valores nominais } E \text { e } \Sigma \text { no intervalo de } \\ Y_{t}^{k}(D, E, \Sigma) & \text { Compo }[0, k] \\ & \text { valo de tempo }[t, k] \\ Y_{t}^{k}(D, B, \Psi) & \text { Covariância do erro de estimação para os valores reais } B \text { e } \Psi \text { no intervalo } \\ & \text { de tempo }[t, k] \\ Y^{k}(D, \cdot, \cdot) & \text { Covariância do erro de estimação no intervalo de tempo }[0, k] \\ \sigma_{1}(A) & \text { Raio espectral de uma matriz A }\end{array}$




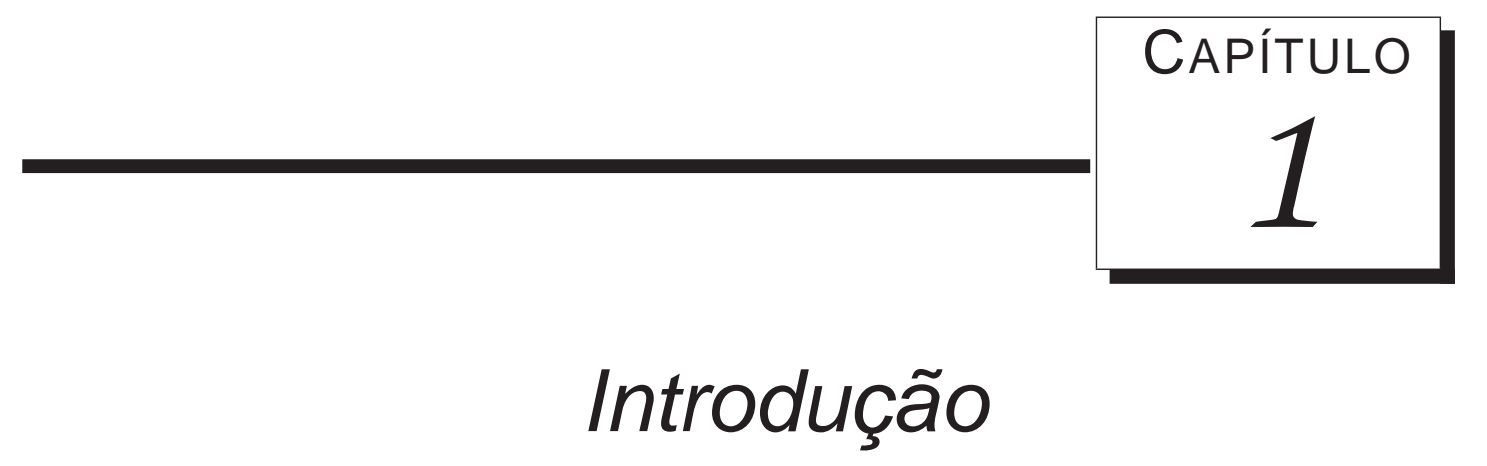

Neste capítulo, apresentamos o cenário de estudo no qual desenvolvemos o trabalho, descrevemos as motivações e estratégias na elaboração dos principais resultados.

Sistemas de controle vêm, há décadas, empregando filtros lineares, também chamados de estimadores lineares ou observadores lineares de estado. Estes filtros são recursivos, apresentando uma dinâmica linear que se baseia na dinâmica da planta e em alguns parâmetros ajustados para, por exemplo, garantir erro de estimação limitado, ou erro de estimação ótimo. Em particular, a teoria de filtragem ótima vem ganhando destaque em diversas áreas de pesquisa, o que a torna cada vez mais relevante. Desde o desenvolvimento do filtro de Kalman, na década de 60, os problemas tem se tornado cada vez mais complexos e estão presentes em um vasto número de aplicações, dentre as quais, podemos citar aplicações em engenharia, telecomunicações, robótica e processamento de sinais. Dada a importância do assunto, é natural o avanço de pesquisas envolvendo a teoria de filtragem. Destacam-se os estudos envolvendo o filtro de Kalman, que é ótimo na classe dos estimadores lineares, [29], [41], sendo clássicas as referências [2] e [51]. Citam-se também os filtros derivados, filtros $H_{\infty}$, filtros $H_{2}$, [1], [35], filtros robustos, veja por exemplo [31], os filtros de Butterworth, filtros de Chebyshev e filtros elípticos, veja [2]. Os argumentos para concentrar-se em filtragem linear são os da aplicabilidade e facilidade de tratamento matemático.

Várias aplicações de filtros encontram-se disponíveis na literatura de sistemas dinâmicos 
e atualmente a teoria a seu respeito é bastante completa. Este é o caso dos sistemas lineares determinísticos invariantes no tempo (SLD) e mesmo dos sistemas determinísticos variantes no tempo. Contudo, o desenvolvimento de aplicações da teoria de filtragem é menos desenvolvida para sistemas cujos parâmetros sejam alterados de maneira aleatória, como é o caso dos chamados sistemas lineares sujeitos a saltos markovianos (SLSM), que como veremos adiante, são sistemas lineares que apresentam mudanças abruptas de comportamento. As mudanças abruptas apresentadas pelos SLSM são "indexadas" por uma variável $\theta(k)$ e podem ser devidas a perturbações ambientais, falha de um componente ou reparos, alterações em subsistemas conectados, modificações no ponto de operação para uma planta não linear. Exemplos dessas situações podem ser encontrados, por exemplo, em economia, controle aéreo, controle de receptores solares, manipuladores robóticos, estruturas para estações espaciais, etc, [15], [24], [28], [38]. O leitor pode encontrar diversos resultados interessantes sobre SLSM nos livros [25], [21] e [34].

Neste trabalho abordaremos, num primeiro cenário, filtros lineares gerais para sistemas lineares com saltos markovianos (SLSM). Em seguida, consideraremos filtros lineares markovianos, os quais possuem a propriedade de que o estimador é um SLSM, ou seja, no instante de tempo $k$ os ganhos e parâmetros dependem somente de $\theta(k)$ (e não de toda a realização de $\theta$ ) veja [? ]. O estimador linear de mínimo erro médio quadrático desenvolvido em [18], [27], [30], [23] é um dos de maior interesse, pois são ótimos na classe dos sistemas lineares markovianos. Em qualquer um desses filtros, a matriz de covariância do erro de estimação forma um processo estocástico, aqui denotado por $Y(k)$. Alguns aspectos relevantes do processo de erro são ainda largamente inexplorados. Destaca-se que a autora não tem conhecimento sobre trabalhos anteriores que tenham considerado uma caracterização da positividade uniforme de $Y(k)$ em termos da estrutura do SLSM em tempo discreto. Igualmente, não há resultados sobre estabilidade de filtros; estabilidade é um tema relevante, uma vez que em situações práticas filtros instáveis podem causar a divergência da covariância do erro de estimação, mesmo com uma mudança infinitesimal na estatística do ruído. Sucintamente, estabilidade exige que a covariância do erro seja limitada, mesmo quando há perturbações no sistema, como descreveremos adiante.

Alguns resultados preliminares estão apresentados em um artigo de conferência [39], no entanto, naquele trabalho abordamos somente estabilidade do filtro de Kalman no contexto dos SLSM com cadeia ergódica e com perturbação apenas na condição inicial, bem mais simples de tratar; resultado análogo para SLSM a tempo contínuo encontram-se em [45]. A 
estabilidade do filtro de Kalman para sistemas lineares variantes no tempo possui um estudo mais amplo, veja [8], [46], [47] e [50]. Vale ressaltar que [9] contém resultados sobre a existência de limitantes superiores para o processo de erro para SLSM (sem as perturbações nas matrizes do processo de ruído aditivo), consequentemente, a estabilidade não é abordada.

Levando em conta o exposto acima, o trabalho aqui apresentado, aborda dois temas centrais, a positividade de $Y$ e a estabilidade do estimador. Destaca-se também o conceito de alcançabilidade média (M-alcançabilidade), que é uma das principais condições de trabalho. Apresentamos a seguir uma descrição um pouco mais detalhada dos resultados e da abordagem utilizada.

\subsection{Terminologia}

Considere um filtro na forma

$$
\hat{x}(k+1)=A_{\theta(k)} \hat{x}(k)+G(k)\left[y(k)-C_{\theta(k)} \hat{x}(k)\right],
$$

sendo $y(k)$ a variável observada (medida a partir do sistema real, geralmente usando sensores) e $\hat{x}(k)$ a estimativa para a variável $x(k)$, que é uma componente do estado de um SLSM. A variável $\theta(k)$ é o estado de uma cadeia de Markov homogênea no tempo, e cada vez que esta variável assume um valor, por exemplo $\theta(k)=i$, então temos $A_{\theta(k)}=A_{i} \mathrm{e}$ $C_{\theta(k)}=C_{i}$, sendo $A_{i}$ e $C_{i}$ conhecidos, tomadas de um conjunto de matrizes. A matriz $G(k)$ é chamada de ganho do filtro, geralmente projetada para atender algum critério sobre o erro de estimação.

Em aplicações reais de controle, muitas vezes acabamos projetando o filtro com base nos parâmetros $E, \Sigma$ da planta e acabamos operando em condições diferentes, com parâmetros $B, \Psi$ substituindo $E, \Sigma$. Por exemplo, isso ocorre quando a planta tem uma variação inesperada durante a operação (possivelmente por envelhecimento), ou quando o projeto de $G(k)$ envolve erros numéricos (como é comum em computadores). O ganho não é recalculado. Em cenários como este, é comum que a covariância do erro de estimação $Y^{k}(B, \Psi)=\mathcal{E}\left\{(x-\hat{x})(x-\hat{x})^{\prime}\right\}$, aqui também denomidada processo de erro, apresente rápida divergência dos valores verdadeiros.

Também estamos interessados na positividade do erro de estimação, em parte pela importância para o estudo de estabilidade. Definimos estes conceitos fundamentais a seguir; a notação $Y^{k}(B, \Psi)$ é usada para deixar claro que se trata do erro de estimação sob os parâmetros 'reais' $B, \Psi$. 
Definição 1.1 (Positividade) Dizemos que o processo de erro é positivo em média quando existem $\lambda>0$ e $K \geq 0$ tais que $\mathcal{E}\left\{Y^{k}(B, \Psi)\right\}-\lambda I$ é uma matriz positiva definida, $k \geq K$.

Definição 1.2 (Estabilidade) Dizemos que um filtro é estável se, para quaisquer $\Psi$ e $B$ existe uma matriz $P$ tal que $\mathcal{E}\left\{Y^{k}(\Psi, B)\right\}-P$ é uma matriz negativa definitiva.

\subsection{Abordagem}

A abordagem adotada neste trabalho é descrita a seguir. Introduzimos o conceito de M-alcançabilidade, o qual envolve uma condição de positividade do gramiano. Em seguida construímos um conjunto de matrizes $\mathcal{R}_{\ell, i}$, denominado matrizes de alcançabilidade e estabelecemos condições para um teste de completude de posto. A abordagem, nesta parte do trabalho, é semelhante a encontrada por exemplo em [10], [11] e [12], referente a observabilidade fraca. Mostram-se que o teste do posto caracteriza a positividade do gramiano de alcançabilidade, Teorema 1. Em seguida, estudamos a positividade da covariância de estado e covariância do erro de estimação no cenário de SLSM e filtros lineares gerais. De fato, provamos que alcançabilidade é uma condição necessária e suficiciente para que o segundo momento condicionado de $x(k), \mathcal{E}\left\{x(k) x(k)^{\prime} \mid \theta(k)=i\right\}$ seja limitado inferiormente por $\beta I$ para algum escalar positivo $\beta$, para estados de Markov recorrentes e $k$ suficientemente grande, como apresentado na Proposição 4.1. Isto implica na positividade de $\mathcal{E}\left\{x(k) x(k)^{\prime}\right\}$, o que significa que o ruído excita, em média, todos os estados do sistema. O resultado é então estendido para o processo de erro do filtro $Y(k)$, estabelecendo no Corolários 5.2 e Corolário 5.3 que $\mathcal{E}\{Y(k)\}$ é positivo para $k$ grande o suficiente, com a interpretação de que a estimativa nunca é precisa (não há componente ou projeção que coincida com $x$ ).

A análise de estabilidade é realizada para a subclasse de sistemas lineares composta de filtros lineares markovianos. Com o objetivo de consolidar uma condição de estabilidade, ajusta-se a condição de alcançabilidade requerendo a positividade de $\mathcal{E}\left\{x(k) x(k)^{\prime} \mid \theta(k)=i\right\}$ para todo $i$ (não somente para estados de Markov recorrentes $i$ ). Vale ressaltar que esta condição pode ser testada numericamente, como oportunamente explicado e é obviamente recorrente da alcançabilidade quando a cadeia de Markov é ergódica.

A análise de estabilidade é realizada por um método direto, através da avaliação e comparação do processo de erro com e sem perturbações nas matrizes de covariância do ruído. Assumimos que o o filtro seja calculado para valores nominais, o qual é caracterizado por certas matrizes de covariância $E_{i}$ e $\Sigma$, em substituição dos valores reais do sistema, $B_{i}$ e 
$\Psi$, respectivamente. Adicionalmente incluímos a hipótese de que a covariância do erro de estimação nominal no instante de tempo $k$, aqui denotada por $Y^{k}(E, \Sigma), k \geq 0$ tenha valor esperado limitado, $\mathcal{E}\left\{Y^{k}(E, \Sigma)\right\} \leq \bar{P}$, uma condição suficiente para esta hipótese seja verdadeira é a detetabilidade estocástica do sistema, veja [6]. Mostra-se de forma construtiva que M-alcançabilidade e a limitação de $\mathcal{E}\left\{Y^{k}(E, \Sigma)\right\} \leq \bar{P}$ são condições suficientes para que exista um limitante superior uniforme no tempo para $\mathcal{E}\left\{Y^{k}(B, \Psi)\right\}$. A maior dificuldade encontrada está na obtenção da uniformidade na limitação do processo de erro. A construção da prova exigiu a introdução de uma recursão no valor esperado, veja Lema 6.1, e na comparação e ordenação de valores de $Y(k)$ envolvendo matrizes de ruído que excitam todas as direções do sistema, Corolário 6.1 e Lema 6.2. O resultado de estabilidade está apresentado no Teorema 3 e Corolário 6.2.

\subsection{Interpretação física e detalhes técnicos}

A interpretação física que norteia as construções das provas apresentadas neste trabalho envolve as relações entre o comportamento da variável de estado e a evolução do estimador. Quando a variável de estado $x$ é completamente ruidosa, não é possível que um filtro linear elimine ruído, logo a covariância do erro de estimação também torna-se ruidosa.

A excitação por ruídos completos é fundamental para revelar qualquer dinâmica instável durante o desenvolvimento do projeto do filtro - se existir uma dinâmica instável, $Y(k)$ cresce indefinidamente, evidenciando a instabilidade do estimador já durante o projeto. Assim, não haveriam dinâmicas instáveis ocultas (que pode acontecer com SLSM que não são M-alcansáveis).

Embora a interpretação dos principais resultados sejam relativamente simples, como descrito acima, os detalhes técnicos e as avaliações necessárias são complexas. As principais dificuldades são as expostas a seguir. Por um lado, como não estamos exigindo ergodicidade da cadeia de Markov, a existência de estados transientes e diferentes conjuntos irredutíveis de estados recorrentes faz com que a análise e notação sejam inevitavelmente complexas. As principais dificuldades técnicas, no entanto, vem do fato de que estamos considerando perturbações no ruído aditivo da variável de estado $x$. Isto exige que busquemos positividade em sentido uniforme do valor esperado do processo de erro, ambos com relação ao estado $x$, no sentido de que exista $\gamma$ satisfazendo $\mathcal{E}\{Y(k) \mid \theta(k)\}>\gamma I$ ( $\gamma$ é uniforme para todos os valores de $k \geq K_{1}$ para um certo $K_{1}$ ). Isto torna algumas passagens mais complexas que em casos nos quais não é necessário que exista uniformidade. Um exemplo é o Teo- 
rema 2, no qual comparamos $Y \operatorname{com} X$, generalizando a conhecido fato de que o filtro não é capaz de eliminar ruído, mostrando que erros de estimação pequenos implicam em erros pequenos na variável $x$, ou mais precisamente, para qualquer $\epsilon \geq 0$, se $v^{\prime} Y(k) v<\epsilon\|v\|^{2}$ então $v^{\prime} X(k) v<h_{k}(\epsilon)\|v\|^{2}$, onde a função $h_{k}$ é $O\left(\epsilon^{\frac{1}{2^{k}}}\right)$, referindo-se a notação de Landau. Surgem também dificuldades técnicas quando comparamos o processo de erro (sob perturbações e sem perturbações), uma vez que podemos compará-los somente em valor esperado condicionado, exigindo a criação de uma recursão para o valor esperado condicionado do processo do erro, como no Lema 6.1. Este resultado nos permite relacionar, algebricamente, o conceito de M-alcançabilidade com a limitação do processo de erro, estabelecendo a estabilidade, Teorema 3 e Corolário 6.2. Este resultado é válido somente para filtros markovianos, pelas razões expostas na Observação 4.

\subsection{Organização do texto}

O texto está organizado da seguinte forma: o Capítulo 2 apresenta as notações utilizadas ao longo do texto, algumas hipóteses e definições, bem como a base da teoria de SLSM e filtragem. O objetivo deste capítulo, além de apresentar a teoria básica, é padronizar e estabelecer foco para o entendimento sobre o apresentado posteriormente. Este capítulo não apresenta nenhum resultado novo sobre a teoria, apenas detalha pontos relevantes já conhecidos e que se fazem necessários no trabalho.

O Capítulo 3 introduz a noção de gramiano de alcançabilidade e o conjunto das matrizes de alcançabilidade. Este capítulo também apresenta alguns resultados auxiliares envolvendo o núcleo das matrizes de alcançabilidade, os quais são utilizados no principal resultado do capítulo, o Teorema do posto completo, que fornece um teste para alcançabilidade média.

Com o objetivo de obter resultados de positividade, introduzimos no Capítulo 4 matrizes similares às matrizes de alcançabilidade, desenvolvemos propriedades de ordenção, caracterizamos a relação entre alcançabilidade e segundo momento do estado e provamos que alcançabilidade média é uma condição necessária e suficiente para que a matriz de covariância do segundo momento seja positiva em valor esperado.

A positividade do processo de erro é de grande interesse neste estudo, sendo apresentada no Capítulo 5. Em virtude dos resultados obtidos não serem extensões diretas dos resultados dos capítulos anteriores, mas serem desenvolvidos por construção, optou-se pela apresentação de resultados de ordenção para realizações da cadeia de Markov e em seguida, extendemos o resultado para o caso mais geral, M-alcançabilidade é condição suficiente para 
positividade do valor esperado do processo de erro, Corolário 5.2 e Corolário 5.3.

Desenvolvemos, finalmente, os resultados de estabilidade no Capítulo 6. A estratégia para obter uma prova de que M-alcançabilidade é condição suficiente para estabilidade está na manipulação de limitantes inferiores e superiores do processo de erro. Construímos um limitante superior uniforme para o processo de erro real, este procedimento exige a utilização de um resultado envolvendo uma espécie de recursão do valor esperado da covariância do erro de estimação, Lema 6.1. Enfim, mostramos que M-alcançabilidade é condição suficiente para estabilidade de filtros markovianos no contexto de cadeias de Markov ergódicas. O Capítulo 6 também ilustra os resultados obtidos, a partir da apresentação e discussão de exemplos.

As conclusões sobre o trabalho desenvolvido, com o destaque de suas principais inovações, são discutidas ao longo do Capítulo 7. 


\section{CAPÍTULO}

\section{Notações, definições e resultados preliminares}

Neste capítulo, apresentamos resultados básicos a serem utilizados no decorrer do texto. O critério para a inclusão ou não de cada um destes neste capítulo baseou-se na importância de cada um para compreensão do estudo. Apresentamos provas para aqueles que não são facilmente encontrados na literatura ou que de alguma forma tenha sido modificado para se enquadrar ao trabalho. Apresentamos também provas para os resultados cujas demonstrações trazem algum argumento técnico que seja relevante em algum ponto do texto. O leitor interessado somente nas contribuições inéditas do trabalho poderia omitir a leitura deste capítulo.

\subsection{Notações}

Nesta seção introduzimos as notações utilizadas no decorrer do texto. Algumas notações adicionais serão acrescentadas posteriormente de acordo com a necessidade de adequação ao contexto ou visando o melhor entendimento do leitor.

Denota-se o espaço euclidiano n-dimensional por $\mathbb{R}^{n}, \mathbb{R}_{+}$o conjunto dos números reais positivos e $\mathbb{Z}_{+}$o conjunto dos números inteiros. $\mathcal{M}^{r, s}$ (respectivamente $\mathcal{M}^{r}$ ) o espaço linear normado formado por todas as matrizes reais $r \times s$ (respectivamente $r \times r$ ). As- 
suma $\mathcal{M}^{r 0}\left(\mathcal{M}^{r+}\right)$ o cone convexo fechado $\left\{U \in \mathcal{M}^{r} \mid U=U^{\prime} \geq 0\right\}$, (o cone aberto $\left.\left\{U \in \mathcal{M}^{r} \mid U=U^{\prime}>0\right\}\right)$, onde $U^{\prime}$ denota a matriz transposta de $U ; U-V \geq 0(U-V>0)$ significa $U-V \in \mathcal{M}^{n 0}\left(U-V \in \mathcal{M}^{n+}\right) . \mathcal{N}^{N, r, s}=\left\{U=\left(U_{1}, U_{2}, \ldots, U_{N}\right)\right\}, N \in \mathbb{Z}_{+}$, denota o espaço linear normado formado por sequências de matrizes reais $U_{i} \in \mathcal{M}^{r, s}, i \in$ $\mathcal{S}=\{1,2, \ldots, N\}$, tal que para $U, V \in \mathcal{N}^{N, r, s}, U+V=\left\{U_{i}+V_{i}, i \in \mathcal{S}\right\}, U \geq V$ denota $U_{i} \geq V_{i}$ para todo $i \in \mathcal{S}$ e similarmente para qualquer operação envolvendo termos pertencentes a $\mathcal{N}^{N, r, s}$. Quando o conjunto $\mathcal{N}^{N, r}$ é formado por $U_{i} \in \mathcal{M}^{r 0}\left(U_{i} \in \mathcal{M}^{r+}\right)$ para todo $i=1,2, \ldots N$, denotamos $\mathcal{N}^{N, r 0}\left(\mathcal{N}^{N, r+}\right) . \mathcal{E}\{\cdot\}$ denota o valor esperado de uma variável aleatória, $\mathcal{P}(\cdot)$ uma medida de probabilidade, $I$ denota a matriz identidade de dimensão apropriada e $\mathbb{I}=\{I, I, \ldots, I\} \in \mathcal{N}^{N, r}$. O símbolo $\|\cdot\|$ denota todas as normas sub multiplicativas de vetores e matrizes. $1_{\{\cdot\}}$ é a função indicadora. Denotamos a cardinalidade de um conjunto $A$ por $\operatorname{Card}(A)$.

A Notação do $O$ maíusculo (A letra $O$ é utilizada devido ao fato da taxa de crescimento de uma função ser denominada ordem), conhecida como símbolo de Landau, é um simbolismo utilizado em ciência da computação e matemática para descrever o comportamento assintótico de funções. Basicamente, informa o quão rápido uma função cresce ou declina. Por exemplo, poderíamos dizer que $f(n)$ cresce com ordem $n^{2}$ e escrever $f(n)=O\left(n^{2}\right)$, veja [5].

\subsubsection{Propriedades usuais do valor esperado}

Destacamos aqui algumas propriedades usuais de valor esperado que serão consideradas no decorrer do texto.

Para quaisquer variáveis aleatórias $A, B, C \in \mathcal{M}^{r, s}$, tem-se as seguintes propriedades para o valor esperado:

1) Se $A \geq B$, então $\mathcal{E}\{A\} \geq \mathcal{E}\{B\}$.

2) Se $A \geq B$, então $\mathcal{E}\left\{C A C^{\prime}\right\} \geq \mathcal{E}\left\{C B C^{\prime}\right\}$.

3) Linearidade : $\mathcal{E}\{(\alpha A+\beta B) \mid C\}=\alpha \mathcal{E}\{A \mid C\}+\beta \mathcal{E}\{B \mid C\}, \alpha, \beta \in \mathbb{R}$.

4) $\mathcal{E}\{A\}=\mathcal{E}\{\mathcal{E}\{A \mid B\}\}$.

Para SLSM, como veremos nos próximos capítulos, o aparecimento de termos com comportamento estocástico, induz a necessidade de introduzir ferramentas da teoria de probabilidades para obtenção de conclusões consideradas em termos do valor esperado. Faremos 


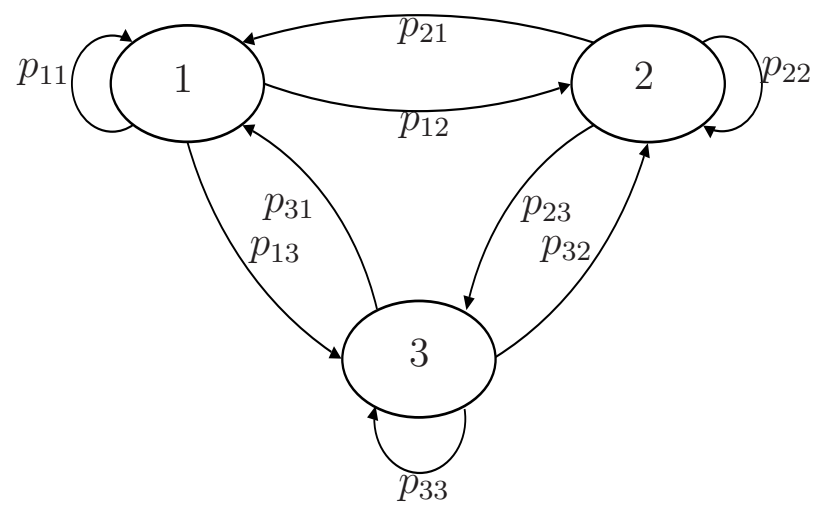

Figura 2.1: Representação da cadeia de Markov com três estados.

uso das propriedades descritas acima de forma indiscriminada, referenciando-as somente em casos nos quais o resultado não seja direto.

\subsection{Cadeia de Markov}

Recentemente os SLSM tem atraído uma atenção considerável, em parte devido ao fato de que fornecem modelos convenientes para aplicações que se caracterizam por mudanças bruscas de comportamento, como frequentemente encontrados em controle de rede e em outros campos, veja por exemplo [23], [34], [37] e [38].

Para melhor entender os sistemas lineares com saltos markovianos, considere um sistema que apresenta mais de um modo de operação, como uma sub-classe de sistemas lineares chaveados, quando as comutações formam um processo estocástico. Sistemas como os descritos acima, alteram seu modo de operação de acordo com uma cadeia de Markov, isto é, a probabilidade deste mover-se de um estado a outro depende unicamente do seu modo atual. Considere ainda que sejam conhecidas todas estas probabilidades de transição do estado $i$ para $j$. A Figura 2.1 traz a representação de um sistema obedecendo uma cadeia de Markov com três modos.

Formalmente, considera-se $\Omega$ um espaço amostral e um processo estocástico $\theta=$ $\left[\theta_{n}, n \in \mathbb{N}\right]$ com espaço de estados enumerável $\mathcal{S}$, isto é, para cada $n \in \mathbb{N}$ e $\omega \in \Omega, \theta_{n}(\omega)$ é um elemento de $\mathcal{S}$, veja [4].

Definição 2.1 O processo estocástico $\theta=\left[\theta_{n} \mid n \in \mathbb{N}\right]$ é chamado uma cadeia de Markov desde que

$$
\mathcal{P}\left(\theta_{n+1}=j \mid \theta_{0}, \ldots, \theta_{n}\right)=\mathcal{P}\left(\theta_{n+1}=j \mid \theta_{n}\right), \forall j \in \mathcal{S}, n \in \mathbb{N}
$$


Denota-se uma cadeia de Markov por $\Theta=\{\theta(k), k \geq 0\}$. Neste trabalho consideramos o espaço de estado tomando valores no conjunto finito $\mathcal{S}$.

Para cada estado existe uma probabilidade, as quais podem ser dispostas em um vetor $\pi$, denominado vetor de probabilidades de estados (para distingui-las das probabilidades de transição). A conexão com a matriz de probabilidade é como segue

$$
\pi(0)=\pi_{0} \text { e } \pi(r)=\pi(r-1) P, \quad 1 \leq r \leq N
$$

onde $P=p_{i j}=\mathcal{P}\{\theta(k+1)=j \mid \theta(k)=i\}$ é a matriz de probabilidade de transição da cadeia de Markov. Dizemos que uma cadeia de Markov é homogênea quando $\mathcal{P}(\theta(k+1)=$ $j \mid \theta(k)=i$ ) é independente de $k$, ou seja, as probabilidades de transição não mudam ao longo do tempo.

\subsubsection{Propriedades da cadeia de Markov}

Considere a cadeia de Markov aperiódica discreta no tempo $\Theta$. Assume-se, sem perda de generalidades, que a matriz de probabilidade de transição assume a seguinte forma, veja [4, Proposition 3.8, Theorem 3.14],

$$
P=\left[\begin{array}{ccccc}
P_{1} & & & & \\
& P_{2} & & & \\
& & \ddots & & \\
& & & P_{r} & \\
& & & O_{r} & Q
\end{array}\right] .
$$

Denota-se por $\mathcal{S}_{\ell}, \ell=1,2, \ldots, r$ os conjuntos de estados de Markov correspondentes a cada matriz de probabilidade $P_{\ell}$ e define-se $R_{P}=\bigcup_{\ell=1}^{r} \mathcal{S}_{\ell}, \operatorname{card}\left(\mathcal{S}_{\ell}\right)=N_{\ell}$ e $\operatorname{card}\left(R_{P}\right)=\bar{N}$. É conhecido que cada $\mathcal{S}_{\ell}$ é um conjunto irredutível fechado de estados recorrentes e desde que a cadeia seja aperiódica, pode-se mostrar que $P_{\ell}$ é a matriz de probabilidade de uma cadeia de Markov ergódica. Além disso, as distribuições de estado para uma cadeia ergódica possuem limitantes na forma $\iota<\mathcal{P}(\theta(k))<\kappa, k \geq K_{0}$, para algum $0<\kappa \leq 1,0<\iota<\kappa$, e $K_{0} \in \mathbb{Z}_{+}$(o qual geralmente depende de $\iota, \kappa$ ), de forma que estes limitantes sejam válidos para cada $\mathcal{S}_{\ell}$. Esta propriedade pode ser facilmente extendida para $\Theta$, levando ao resultado na Proposição 2.1 .

Para dar contexto ao resultado seguinte, destacamos que parte do estudo é feita em pe- 
ríodos de tempo finito, motivando a caracterização da distribuição da cadeia de Markov em um intervalo de tempo específico. O resultado é uma adaptação de [4, Theorem 3.2].

Proposição 2.1 Existem $0<\kappa \leq 1,0<\iota<\kappa e K_{0} \in \mathbb{Z}_{+}$tais que, para cada $1 \leq \ell \leq r$,

$$
\iota \mathcal{P}\left(\theta(N) \in \mathcal{S}_{\ell}\right) \leq \pi_{i}(k) \leq \kappa \mathcal{P}\left(\theta(N) \in \mathcal{S}_{\ell}\right), \forall k \geq K_{0}+N, i \in S_{\ell}
$$

Para $K_{0}$ como considerado acima, definimos a seguinte constante, a qual será empregada em todo o texto,

$$
K_{1}=K_{0}+n^{2} \bar{N}+N
$$

Introduzimos a seguinte noção de estados alcançáveis.

Definição 2.2 Dado $\pi(0)=\pi$, considere o conjunto

$$
R_{\pi}=\left\{i \in R_{P} \mid \exists j_{0}, \ldots, j_{m} \text { e } p_{j_{0} j_{1}}, p_{j_{1} j_{2}}, \ldots, p_{j_{m} i}>0, \pi_{j_{0}}>0\right\}
$$

de estados $\theta$ que podem ser alcançados a partir de estados iniciais com probabilidade positiva.

\subsection{Sistemas lineares com saltos markovianos}

Nesta seção, apresentamos e descrevemos brevemente algumas das propriedades dos sistemas lineares com saltos markovianos, dos quais tratamos no trabalho. Consideram-se SLSMs em termos discretos e de dimensão finita.

Os SLSMs são sistemas cuja dinâmica muda abruptamente em instantes de tempo indeterminados e comportam-se como sistemas lineares nos demais instantes. As alterações repentinas na dinâmica do sistema são denominados saltos e referem-se a mudanças em parâmetros do sistema, as quais ocorrem de acordo com uma cadeia de Markov subjacente. Desta forma, os SLSM são sistemas dinâmicos estocásticos, cuja propriedade característica é que cada salto não conserva a memória dos instantes de tempo decorridos e somente o estado no instante atual pode influênciar no comportamento do sistema no instante seguinte. Neste Trabalho, abordamos exclusivamente o caso em que o processo de Markov subjacente percorre um conjunto de estados discreto finito. 
A motivação para o estudo deste tipo de sistema decorre do fato de que grande parte dos sistemas de controle são baseados em modelos matemáticos de processos a serem controlados, os quais podem ser descritos, por exemplo, por modelos de sistemas lineares invarantes no tempo, mas um grande número está sujeito a mudanças incertas em sua dinâmica. Se essa mudança é abrupta, tendo pouca influência no comportamento do sistema, uma análise clássica de sensibilidade do sistema pode fornecer uma avaliação adequada dos efeitos. No entanto, quando as variações causadas pelas mudanças transformam o comportamento do sistema de forma significativa, é preferível um modelo estocático que apresente a indicação da probabilidade relativa dos vários cenários possíveis. Para ilustrar a situação, considere um sistema dinâmico que é, em um determinado momento, bem representado por um modelo $\Phi_{1}$. Suponha que este modelo esteja sujeito a mudanças que torne este modelo, após um período de tempo, em um modelo diferente, digamos $\Phi_{2}$. Com este mesmo raciocínio podemos considerar que o sistema esteja sujeito a uma série de possíveis mudanças qualitativas que transformem o sistema em outro sistema pertencente a um conjunto finito de modelos $\left\{\Phi_{1}, \Phi_{2}, \ldots, \Phi_{N}\right\}$. Podemos associar cada um dos modelos a um modo de operação do sistema ou a apenas saltos de um modo para outro, ou que existe uma transição entre os modos. Assumimos neste trabalho que os saltos do sistema descrito acima evoluem de acordo com uma cadeia de Markov, isto é, dado que em um certo instante de tempo $k$ o sistema permanece em um estado $i$, sabe-se que a probabilidade de salto para qualquer um dos outros modos, e também a probabilidade de permanecer no modo $i$, depende somente do modo de operação no instante de tempo atual. Tais sistemas são denominados sistemas lineares com saltos markovianos e o estado de Markov (ou modo de operação) será denotado por $\theta(k)$. Para uma caracterirazação mais completa veja [24].

A seguir, apresentamos a formulação do sistema linear a tempo discreto com saltos markovianos que consideraremos no trabalho.

Considere que a cadeia de Markov homogênea $\Theta=\{\theta(k) \mid k \geq 0\}$ assume valores em $\mathcal{S}$, sendo caracterizada pela matriz de probabilidades $P=\left[p_{i j}, i, j \in S\right]$, com distribuição de probabilidade inicial $\mathcal{P}(\theta(k)=i)=\pi_{i}(k), i \in S$.

Seja $A_{i} \in \mathcal{H}^{N, n}, B_{i} \in \mathcal{H}^{N, n, q}, C_{i} \in \mathcal{H}^{N, r, n}$ e $D_{i} \in \mathcal{H}^{N, r, q}$. Consideramos o SLSM 
definido em um espaço de probabilidade $\left(\Omega, \mathfrak{F}, \mathfrak{F}_{k}, \mathcal{P}\right)$ como sendo:

$$
\Phi_{M}:\left\{\begin{aligned}
x(k+1) & =A_{\theta(k)} x(k)+B_{\theta(k)} w(k), \\
y(k) & =C_{\theta(k)} x(k)+D_{\theta(k)} v(k), k \geq 0, \\
x(0) & =x_{0}, \theta(0)=\theta_{0},
\end{aligned}\right.
$$

assume-se $D_{i} D_{i}^{\prime}>0$ (ruído não singular). O par $(x(k), \theta(k))$ é a variável de estado, onde $x(k) \in \mathbb{R}^{n}$ é a variável de estado contínua, com $x(0)$ uma variável aleatória de média nula satisfazendo $\mathcal{E}\left\{x(0) x(0)^{\prime}\right\}=\Psi$ e $\theta(k) \in \mathcal{S}$ é a variável de estado discreta. $y(k) \in \mathbb{R}^{r}$ é a variável de observação, $w(k) \in \mathbb{R}^{p}$ e $v(k) \in \mathbb{R}^{q}$ formam ruídos estocásticos estacionários independentes, com média nula e satisfazendo $\mathcal{E}\left\{w(k) w(k)^{\prime}\right\}=I, \mathcal{E}\left\{v(k) v(k)^{\prime}\right\}=I$. Assume-se também que $x(0),\{w(k)\}$ e $\{v(k)\}$ são mutuamente independentes. Assume-se observação das variáveis de saída e de estado discreto, ou seja, as informações disponíveis no instante de tempo $k \geq 0$ são dadas por $\mathcal{F}_{k}=\{y(0), \theta(0), \ldots, y(k), \theta(k)\}$. Denota-se $\Theta_{k}=\left\{x_{0}, \theta(0), \ldots, \theta(k)\right\}$. Para demais aspectos do SLSM, referênciam-se [15], [24], [28], [21], [38].

São diversas as aplicações para SLSM, dentre as quais citamos a área de aeronáutica [3], modelos macroeconômicos [32] e receptores térmicos [49]. Muitos resultados teóricos estão presentes na literatura de SLSM, dentre os quais destacamos [16], [17], [19], [22],[28], [21], [33], [43], [44] e [48]. Aspectos sobre como os SLSM generalizam sistemas lineares discretos podem ser encontrados em [13] e [37].

\subsubsection{Segundo momento de $x(k)$}

O segundo momento do estado $x(k)$ está ligado às matrizes de M-alcançabilidade, como veremos no Capítulo 3, sendo fundamental no desenvolvimento de resultados de positividade relacionados à estabilidade.

Introduzimos o processo estocástico no espaço $\mathcal{M}^{n}$ dado por

$$
\begin{aligned}
X(k+1) & =A_{\theta(k)} X(k) A_{\theta(k)}^{\prime}+B_{\theta(k)} B_{\theta(k)}^{\prime}, \\
X(t) & =\Psi, \quad k \geq t \geq 0 .
\end{aligned}
$$

Com o objetivo de enfatizar a dependência das variáveis $B$ e $\Psi$, denotamos $X(k)$ por $X_{t}^{k}(\Psi, B)$ ou por $X^{k}(\Psi, B)$ quando $t=0$. Os sistemas $X(k)$ e $x(k)$ estão ralacionados como abaixo, veja [9, Proposition 1]. 
Proposição 2.2 Considere o sistema $\Phi_{M}$ em (2.4) e X(k) definido em (2.5). Então

$$
\mathcal{E}\left\{x(k) x^{\prime}(k) \mid \Theta_{k}\right\}=X^{k}(B, \Psi) .
$$

\subsection{Filtros lineares}

Problemas de filtragem são de interesse não só por causa de seu grande número de aplicações, mas também por ser o principal passo para estudar problemas com observações parciais sobre a variável de estado. Atualmente existem diversos estudos envolvendo filtragem. Entre as ferramentas matemáticas significativas que podem ser utilizadas para a estimativa de processos estocásticos, uma das mais conhecidas e frequentemente utilizadas é o filtro de Kalman. Com poucas ferramentas computacionais, o filtro de Kalman é atualmente muito utilizado.

Neste trabalho, consideramos filtros lineares dados por $\hat{x}(0)=0 \mathrm{e}$

$$
\hat{x}(k+1)=A_{\theta(k)} \hat{x}(k)+G(k)\left[y(k)-C_{\theta(k)} \hat{x}(k)\right],
$$

assume-se que o ganho do filtro é uma função do tempo e de $\Theta_{k}$, isto é,

$$
G(k)=g(k, \hat{x}(0), \theta(0), \ldots, \theta(k))
$$

para alguma função $g$ (que pode ser trivial em alguns de seus argumentos).

Definimos $\tilde{x}(k)=x(k)-\hat{x}(k), k \geq 0$ e obtemos de (2.4) e (2.6) que

$$
\tilde{x}(k+1)=\left(A_{\theta(k)}-G(k) C_{\theta(k)}\right) \tilde{x}(k)+B_{\theta(k)} w(k)-G(k) D_{\theta(k)} v(k) .
$$

A covariância do erro de estimação $Y \in \mathcal{M}^{n}$ é definida por

$$
Y^{k}(D, B, \Psi)=\mathcal{E}\left\{\tilde{x}(k) \tilde{x}^{\prime}(k) \mid \Theta_{k}\right\}
$$

Dados $U \in \mathcal{N}^{N, r, q}$ e $V \in \mathcal{N}^{N, n, p}$, considere

$$
\Upsilon_{k}(U, V)=G(k) U_{\theta(k)} U_{\theta(k)}^{\prime} G^{\prime}(k)+V_{\theta(k)} V_{\theta(k)}^{\prime},
$$

A covariância do erro de estimação $Y^{k}(D, B, \Psi)$ pode ser escrita como na proposição a 
seguir.

\section{Proposição 2.3}

$$
\begin{aligned}
Y(k+1) & =\left(A_{\theta(k)}-G(k) C_{\theta(k)}\right) Y(k)\left(A_{\theta(k)}-G(k) C_{\theta(k)}\right)^{\prime}+\Upsilon_{k}(D, B), \\
Y(t) & =\Psi, k+1 \geq t \geq 0 .
\end{aligned}
$$

Veja [9], [47]. Similarmente a (2.5), denotamos $Y(k)$ por $Y_{t}^{k}(D, B, \Psi)$, ou $Y^{k}(D, B, \Psi)$, quando $t=0$.

Observação 1 Observe que o filtro é definido de forma similar ao utilizado para sistemas lineares variantes no tempo, [7], entretanto note que as informações $\mathcal{F}_{k}$ permitem o cálculo de $Y(k)$ e $G(k)$ no instante de tempo $k$ (implementações off-line, nas quais os ganhos $L(k)$ calculados a priori não são considerados), como em [9]. Note também que as variáveis $Y(k)$ e $G(k)$ estão em função das variáveis aleatórias $\theta(0), \theta(1), \ldots, \theta(k)$, de maneira que formam processos estocásticos, requerendo que as covariâncias sejam estudadas em valor esperado.

Observação $2 \mathrm{Se} B_{i}$ é de posto completo, então o ruído acarreta uma excitação persistente e completa na dinâmica do filtro, note que de (2.10), $Y^{k}(D, B, \Psi) \geq \min _{i}\left(\sigma_{1}\left(B_{i} B_{i}^{\prime}\right)\right) I$. Isto pode levar à divergência de $\mathcal{E}\left\{Y^{k}(D, B, \Psi)\right\}$ (ao passo que seria limitada se $B=\Psi$ ), revelando dinâmicas instáveis.

Em virtude do fato de que as dinâmicas em (2.10) são estocásticas, estudar $\mathcal{E}\{Y(k)\}$ é uma questão bastante complexa. Uma condição bastante óbvia para se obter a limitação de $\mathcal{E}\{Y(k, B, \Psi)\}$ é a de que $\mathcal{E}\{Y(k, E, \Sigma)\}$ seja limitada.

Suposição 1 [Limitação] Existe $\bar{P} \in \mathcal{M}^{n 0}$ tal que $\mathcal{E}\{Y(k)\}<\bar{P}, \forall k \geq 0$.

A homogeneidade da cadeia de Markov é relevante na prova de resultados que exijam translações no tempo, generalizações (noções de uniformidade) e induções no tempo; consequentemente, é necessária a introdução da noção de filtros homogêneos no tempo, conceito similar à homogeneidade da cadeia. Assumimos que o filtro linear é homogêneo no tempo no seguinte sentido.

$$
\begin{aligned}
\mathcal{P}\left(Y^{k}(Y(0)=\Psi) \mid \theta(0)=j,\right. & \theta(k)=i) \\
& =\mathcal{P}\left(Y^{k+t}(Y(t)=\Psi) \mid \theta(t)=j, \theta(k+t)=i\right) .
\end{aligned}
$$




\subsection{Filtros lineares markovianos}

Neste trabalho estudamos também características e propriedades teóricas de filtros markovianos, os quais possuem a propriedade de que o sistema de malha fechada é novamente um SLSM, veja [? ]. Uma desvantagem dos filtros lineares na forma (2.6)-(2.7) é que os ganhos exigem cálculos on-line, que é muitas vezes difícil e restringe aplicações computacionais. Uma maneira de superar essa dificuldade é a de considerar a subclasse de filtros lineares markovianos, cujos ganhos são da forma

$$
G(k)=g(k, \theta(k))
$$

e são selecionados a partir de uma sequência de conjuntos de matrizes $H_{i}(k)$, tomando

$$
G(k)=H_{\theta(k)}(k)
$$

em cada momento $k$. A sequência $H$ é projetada independentemente da realização da cadeia de Markov, com base apenas nas informações disponíveis antes da operação do sistema, como apresentado por exemplo em [26], [? ] e [20].

A covariância do erro de estimação é dada pela solução $Y(k)$ de (2.10). Ao lidar com filtros markovianos, será conveniente expressar $Y(k)$ decomposto em soluções homogêneas e soluções forçadas de (2.13), como segue.

O processo dado por (2.10) satisfaz: $Y_{t}^{t}(0,0, \Psi)=\Psi$,

$$
\begin{aligned}
Y_{t}^{k}(0,0, \Psi) & =\left(A_{\theta(k)}-H_{\theta(k)}(k) C_{\theta(k)}\right) \ldots\left(A_{\theta(t)}-H_{\theta(t)}(t) C_{\theta(t)}\right) \Psi \\
& \times\left(A_{\theta(t)}-H_{\theta(t)}(t) C_{\theta(t)}\right)^{\prime} \ldots\left(A_{\theta(k)}-H_{\theta(k)}(k) C_{\theta(k)}\right)^{\prime}
\end{aligned}
$$

$\mathrm{e}$

$$
Y_{t}^{k}(D, B, \Psi)=Y_{t}^{k}(0,0, \Psi)+\sum_{\ell=t+1}^{k} Y_{\ell}^{k}\left(0,0, \Upsilon_{\ell-1}(D, B)\right)
$$

Dada uma sequência $\theta(0), \theta(1), \ldots, \theta(k), k \geq 0$, tem-se

$$
Y^{k}(D, B, \Psi)=Y_{t}^{k}\left(0,0, Y^{t}(D, B, \Psi)\right)+\sum_{\ell=t+1}^{k} Y_{\ell}^{k}\left(0,0, \Upsilon_{\ell-1}(D, B)\right)
$$


dessa forma, a fim de expressar a recursão $Y(k)$ no intervalo de tempo $[t, k]$, quando conveniente, denotaremos

$$
Y^{k}(D, B, \Psi)=Y_{t}^{k}\left(D, B, Y^{t}(D, B, \Psi)\right), k \geq t>0
$$

Como exemplo de filtro markoviano, citamos o estimador linear de mínimo erro médio quadrático desenvolvido em [23], que são ótimos na classe dos sistemas lineares markovianos. 


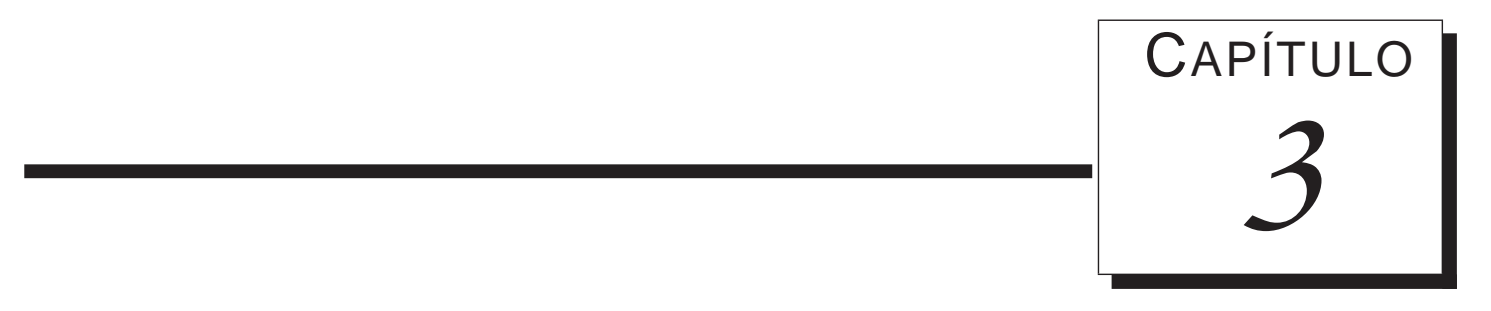

\section{M-alcançabilidade e matrizes de M-alcançabilidade}

Neste capítulo introduzimos o conceito de alcançabilidade média (M-alcançabilidade). Inicialmente apresentamos uma definição de alcançabilidade envolvendo a positividade do gramiano. Em seguida, introduzimos um conjunto de matrizes $R_{\ell, i}, \ell=1,2, \ldots, r, i \in$ $\mathcal{S}_{\ell}$, o qual definimos como sendo o conjunto das matrizes de alcançabilidade, o qual se assemelha ao conjunto de matrizes de alcançabilidade de SLD. Resultados relacionados à dimensão de $R_{\ell, i}$ são incluídos. Apresentamos, também, propriedades envolvendo o núcleo das matrizes $R_{\ell, i}$. Enfim, desenvolve-se o principal resultado do capítulo: cada matriz do conjunto de matrizes de M-alcançabilidade é de posto completo se e somente se o sistema é M-alcançável, estabelecendo um paralelo com o teste de M-alcançabilidade para sistemas lineares determinísticos.

\subsection{M-alcançabilidade, gramiano e matrizes de M-alcançabilidade}

Alcançabilidade é um conceito clássico na teoria dos sistemas lineares, todavia não se encontra na literatura o desenvolvimento do conceito para SLSM. Por ser uma propriedade que possibilita controle sobre os estados do sistema (alcançabilidade implica em controlabi- 
lidade, mas a recíproca não é sempre verdadeira. Controlabilidade implica em alcançabilidade somente se as matrizes consideras são inversíveis), surge o questionamento de como a alcançabilidade poderia afetar o processo de erro gerado por estimadores.

Com o objetivo de firmar o conceito de alcançabilidade no contexto de SLSM e na busca por condições que sejam necessárias e/ou suficientes para que se obtenha limitação do processo de erro, apresentamos a seguir, um estudo da noção de M-alcançabilidade.

Considere o Gramiano de alcançabilidade $\mathcal{G}: \mathbb{Z}_{+} \rightarrow \mathcal{M}^{n 0}$ do sistema (2.4) definido por

$$
\mathcal{G}(k)=\sum_{t=0}^{k-1} \Phi(k, t+1) B_{\theta(t)} B_{\theta(t)}^{\prime} \Phi^{\prime}(k, t+1), \theta(k) \in \mathcal{S}, k>t \geq 0,
$$

onde

$$
\Phi(k+1, t)=A_{\theta(k)} A_{\theta(k-1)} \ldots A_{\theta(t)} .
$$

Consideramos o seguinte conceito de alcançabilidade média, o qual é uma adaptação do conceito de alcançabilidade para sistemas lineares variantes no tempo (caso discreto) e possui a interpretação de que um subspaço alcançável é aquele composto por todos os pontos $x_{1}$, em um instante de tempo $t_{1}$, para os quais exista uma entrada (que dependa de $t_{0}, t_{0}+1, \ldots, t_{1}-$ 1) que transfira o estado do ponto inicial $x\left(t_{0}\right)=0$ à $x\left(t_{1}\right)=x_{1}$, veja por exemplo [42].

Definição 3.1 (M-Alcançabilidade) Dizemos que $(A, B, P)$ é M-alcançável quando existem um inteiro $k$ e um escalar $\gamma \in \mathbb{R}_{+}$tais que

$$
\mathcal{E}\{\mathcal{G}(k) \mid \theta(0)=j\} \geq \gamma I, j \in \mathcal{S}
$$

Note que mesmo no cenário de SLSM, é simples exemplificar um caso não alcançável, basta considerar um sistema que não seja infuenciado pela entrada

$$
x(k+1)=A_{\theta(k)} x(k), k \geq 0 .
$$

No entanto, a construção (ou verificação) de um sistema alcançável pode não ser uma tarefa simples. Para tanto, desenvolvemos um resultado que permite testar a M-alcançabilidade.

Considere as seguintes definições. 
Para cada $\ell=1,2, \ldots, r$ e $i \in \mathcal{S}_{\ell}$, definimos

$$
M_{\ell, i}=\sum_{j=0}^{N} p_{j i} B_{j} B_{j}^{\prime} 1_{\left\{j \in \mathcal{S}_{\ell}\right\}}, \quad i \in \mathcal{S}_{\ell}
$$

Definimos o operador $\mathcal{A}_{\ell, i}: \mathcal{N}^{N_{\ell}, n 0} \rightarrow \mathcal{N}^{N_{\ell}, r 0}$, de forma que para $U \in \mathcal{N}^{N_{\ell}, n 0}$,

$$
\mathcal{A}_{\ell, i}(U)=\sum_{j \in \mathcal{S}_{\ell}} p_{j i} A_{j} U_{j} A_{j}^{\prime}, \quad i \in \mathcal{S}_{\ell}
$$

Mostra-se em [22] que $\mathcal{A}$ dado em (3.4) é um operador linear semi-definido positivo. Denota-se $\mathcal{A}^{0}(U)=U$ e para $\mathcal{A}: \mathcal{N}^{N_{\ell}, n 0} \rightarrow \mathcal{N}^{N_{\ell}, n 0}$, definimos $\mathcal{A}^{k}(U)$ recursivamente por $\mathcal{A}^{k}(U)=\mathcal{A}\left(\mathcal{A}^{k-1}(U)\right), k>0$.

Definem-se as coleções de matrizes $R_{\ell, i}(k) \in \mathcal{M}^{n 0}$ por

$$
\begin{aligned}
R_{\ell, i}(k+1) & =\mathcal{A}_{\ell, i}\left(R_{\ell}(k)\right)+M_{\ell, i}, \quad k \geq 0, \quad i \in \mathcal{S}_{\ell} \\
R_{\ell, i}(0) & =0, \quad i \in \mathcal{S}_{\ell}
\end{aligned}
$$

Observe que, com a notação introduzida, temos

$$
R_{\ell}(k+1)=\sum_{t=0}^{k} \mathcal{A}_{\ell}^{t}\left(M_{\ell}\right), \quad k \geq 0 .
$$

Como buscamos um teste de posto para M-Alcançabilidade baseado nas matrizes $R_{\ell}(k)$, é de interesse utilizar a linearidade do operador $\mathcal{A}$ e o Teorema de Cayley-Hamilton para reduzir a dimensão do teste. Os resultados seguintes podem ser obtidos como uma adaptação direta dos resultados em [14, Lemma 3] e [39, Lemma 2].

Lema 3.1 Considere as matrizes definidas em (3.5). Para cada $\ell=1,2 \ldots, r$ e $i \in \mathcal{S}_{\ell}$, temos que

$$
\operatorname{posto}\left(\left[R_{\ell, i}(1) \vdots R_{\ell, i}(2) \vdots \ldots \vdots R_{\ell, i}(k)\right]\right)=n,
$$

para algum $k \geq n^{2} N_{\ell}$, se e somente se

$$
\operatorname{posto}\left(R_{\ell, i}\left(n^{2} N_{\ell}\right)\right)=n
$$

Assumindo este resultado, neste trabalho lidamos com a seguinte definição de matrizes de M-alcançabilidade $\mathcal{R}_{\ell, i}$. 
Definição 3.2 A coleção de matrizes de M-alcançabilidade é dada por,

$$
\mathcal{R}_{\ell, i}=R_{\ell, i}\left(n^{2} N_{\ell}\right), \quad i \in \mathcal{S}_{\ell}, \quad \ell=1,2, \ldots, r
$$

\subsubsection{Propriedades do $\mathcal{K} \operatorname{er}\left\{R_{\ell, i}(k)\right\}$}

Apresentamos agora algumas propriedades do espaço nulo das matrizes $R_{\ell, i}(k)$, as quais serão utilizadas na prova do Teorema 1.

Lema 3.2 Se para algum $1 \leq \ell \leq r$, existem $v \in \mathbb{R}^{n}$ não trivial e $i \in \mathcal{S}_{\ell}$, tais que

$$
\mathcal{K} e r\left\{R_{\ell, i}(k)\right\} \subset \mathcal{K} \operatorname{er}\left\{\Phi(k, t)\left[M_{\ell, i}\right] \Phi^{\prime}(k, t)\right\}
$$

quase certamente (q.c $)^{1}$ para $k \geq t \geq 0$.

Demonstração: Suponha que existam um vetor não trivial $v \in \mathbb{R}^{n}$ e $1 \leq \ell \leq r$, tais que para algum $i \in \mathcal{S}_{\ell}$ e $v \in \mathcal{K} \operatorname{er}\left\{R_{\ell, i}(k)\right\}, k \geq 0$, então por (3.5) segue que

$$
\begin{aligned}
0 & =v^{\prime} R_{\ell, i}(k) v \\
& =v^{\prime}\left(\mathcal{A}_{\ell, i}\left[\sum_{t=0}^{k-1} \mathcal{A}_{\ell}^{t}\left(M_{\ell}\right)\right]+M_{\ell, i}\right) v \\
& =v^{\prime}\left(\mathcal{A}_{\ell, i}\left(M_{\ell}\right)+\mathcal{A}_{\ell, i}\left[\mathcal{A}_{\ell}\left(M_{\ell}\right)\right]+\ldots+\mathcal{A}_{\ell, i}\left[\mathcal{A}_{\ell}^{k-1}\left(M_{\ell}\right)\right]+M_{\ell, i}\right) v \\
& \geq v^{\prime}\left(\mathcal{A}_{\ell, i}\left[\mathcal{A}_{\ell}^{t}\left(M_{\ell}\right)\right]\right) v, \quad t=1,2, \ldots, k-1 .
\end{aligned}
$$

(3.8) leva a

$$
v^{\prime} \in \mathcal{K} e r\left\{\mathcal{A}_{\ell, i}\left[\mathcal{A}_{\ell}^{t}\left(M_{\ell}\right)\right]\right\}
$$

Expandindo $\mathcal{A}_{\ell}^{t}$ obtém-se avaliações da forma

$$
p_{j_{1} i} A_{j_{1}} p_{j_{2} i} A_{j_{2}} \ldots p_{j_{t} i} A_{j_{t}}\left(M_{\ell, i}\right) A_{j_{1}}^{\prime} A_{j_{2}}^{\prime} \ldots A_{j_{t}}^{\prime}
$$

portanto (3.8) implica que

$$
v \in \mathcal{K} e r\left\{p_{j_{1} i} A_{j_{1}} p_{j_{2} j_{1}} A_{j_{2}} \ldots p_{j_{t} j_{t-1}} A_{j_{t}}\left(M_{\ell, j_{t}}\right) A_{j_{1}}^{\prime} A_{j_{2}}^{\prime} \ldots A_{j_{t}}^{\prime}\right\}
$$

\footnotetext{
${ }^{1} P\left(v \in \mathcal{K} \operatorname{er}\left\{\left\{\Phi(k, t)\left[M_{\ell, i}\right] \Phi^{\prime}(k, t)\right\}\right)=1\right.$
} 
onde a coleção de índices $j_{1}, j_{2}, \ldots j_{t}, t=1,2, \ldots, k$ pertence a $\mathcal{S}_{\ell}$. Como $\mathcal{S}_{\ell}$ é um conjunto fechado irredutível, segue que todo valor de probabilidade $p_{j_{t} i}$ é positivo, portanto

$$
v \in \mathcal{K} e r\left\{A_{j_{1}} A_{j_{2}} \ldots A_{j_{t}}\left(M_{\ell, j_{t}}\right) A_{j_{1}}^{\prime} A_{j_{2}}^{\prime} \ldots A_{j_{t}}^{\prime}\right\}, t=1,2, \ldots, k
$$

Logo,

$$
\mathcal{P}\left(v \in \mathcal{K} \operatorname{er}\left\{\Phi(k, t)\left(M_{\ell, j_{t}}\right) \Phi^{\prime}(k, t)\right\}\right)=1, k \geq t \geq 0
$$

O resultado segue tomando $j_{t}=i$.

Corolário 3.1 Considere $R_{\ell}$ como definido em (3.5). Tem-se $\mathcal{K} e r\left\{R_{\ell, j}(k)\right\}=\mathcal{K} \operatorname{er}\left\{R_{\ell, i}(k)\right\}$, para todo $i, j \in \mathcal{S}_{\ell}$ e $k \geq 0$.

Demonstração: Suponha que existam um valor não trivial $\ell \leq r, j \in \mathcal{S}_{\ell}$ e $v \in$ $\mathcal{K} e r\left\{R_{\ell, j}(k)\right\}$, então

$$
0=v^{\prime}\left[\mathcal{A}_{\ell, j}\left(R_{\ell}(k)\right)+M_{\ell, j}\right] v=v^{\prime} M_{\ell, j} v
$$

Segue do Lema 3.2 que

$$
v \in \mathcal{K} e r\left\{\Phi(k, t)\left[\sum_{\ell=1}^{N} p_{\ell j} B_{\ell} B_{\ell}^{\prime} 1_{\left\{\ell \in \mathcal{S}_{\ell}\right\}}\right] \Phi^{\prime}(k, t)\right\}, k \geq t, \text { q.c. }
$$

Como $p_{\ell i}>0$ para todo $i \in \mathcal{S}_{\ell}$, tem-se

$$
v \in \mathcal{K} e r\left\{\Phi(k, t)\left[\sum_{\ell=1}^{N} p_{\ell i} B_{\ell} B_{\ell}^{\prime} 1_{\left\{\ell \in \mathcal{S}_{\ell}\right\}}\right] \Phi^{\prime}(k, t)\right\}, \forall i \in \mathcal{S}_{\ell}
$$

Como consequência

$$
\begin{aligned}
v^{\prime}\left\{\sum _ { t = 0 } ^ { k - 1 } \left(\sum_{\ell=1}^{N} p_{\ell i} A_{\ell_{k-1}}\right.\right. & \sum_{\ell=1}^{N} p_{\ell i} A_{\ell_{k-2}} \ldots \\
& \left.\left.\sum_{\ell=1}^{N} p_{\ell i} A_{\ell_{1}}\left[\sum_{\ell=1}^{N} p_{\ell i} B_{\ell} B_{\ell}^{\prime} 1_{\left\{\ell \in \mathcal{S}_{\ell}\right\}}\right] A_{\ell_{k-1}}^{\prime} A_{\ell_{k-2}}^{\prime} \ldots A_{\ell_{1}}^{\prime}\right)\right\} v=0
\end{aligned}
$$

portanto

$$
0=v^{\prime}\left[\mathcal{A}_{\ell, i}\left(\sum_{t=0}^{k-1} \mathcal{A}_{\ell}^{t}\left(M_{\ell}\right)\right)+M_{\ell, i}\right] v=v^{\prime} R_{\ell, i}(k) v
$$


Logo concluímos que $v \in \mathcal{K} e r\left\{R_{\ell, i}(k)\right\}$, para todo $i \in \mathcal{S}_{\ell}$.

\subsection{O Teorema do posto completo}

Nesta seção, mostramos que o conceito de M-alcançabilidade dado em 3.1 é equivalente à posto completo de $\mathcal{R}_{\ell, i}, i \in \mathcal{S}_{\ell}$. Fornecendo um teste para M-alcançabilidade.

Teorema 1 (Teorema do posto completo) Considere a coleção de matrizes de M-alcançabilidade $\mathcal{R}_{\ell, i}$, em (3.7). $\quad(A, B, P)$ é M-alcançável se e somente se, para cada $\ell=1,2, \ldots, r$, tem-se posto $\left(\mathcal{R}_{\ell, i}\right)=n, i \in \mathcal{S}_{\ell}$.

Demonstração: Para facilitar a escrita dos resultados, nesta prova consideramos

$$
\phi_{t, j}=\Phi\left(n^{2} \bar{N}+2 N, t+1\right) B_{j} B_{j}^{\prime} \Phi^{\prime}\left(n^{2} \bar{N}+2 N, t+1\right),
$$

onde $\Phi$ é como em (3.2). Definimos a função semidefinida positiva

$$
f(v, j)=v^{\prime} \mathcal{E}\left\{\mathcal{G}\left(n^{2} \bar{N}+2 N\right) \mid \theta(0)=j\right\} v, j \in \mathcal{S} .
$$

Observe que da definição do Gramiano de alcançabilidade em (3.1), tem-se

$$
\begin{aligned}
f(v, j) & =v^{\prime} \mathcal{E}\left\{\left[\sum_{t=0}^{n^{2} \bar{N}+2 N-1} \Phi\left(n^{2} \bar{N}+2 N, t+1\right)\right.\right. \\
\left.\left.B_{\theta(t)} B_{\theta(t)}^{\prime} \Phi^{\prime}\left(n^{2} \bar{N}+2 N, t+1\right)\right] \mid \theta(0)=j\right\} v & v^{\prime} \mathcal{E}\left\{\sum_{t=0}^{n^{2} \bar{N}+2 N-1} \phi_{t, \theta(t)} \mid \theta(0)=j\right\} v
\end{aligned}
$$

(Suficiência) Suponha que $(A, B, P)$ não seja M-alcançável, ou seja, para qualquer escalar $\gamma$ e qualquer inteiro $K$ existem $v \in \mathbb{R}^{n}$ e $j \in \mathcal{S}$ tais que

$$
v^{\prime} \mathcal{E}\{\mathcal{G}(K) \mid \theta(0)=j\} v<\gamma\|v\|^{2}
$$

Primeiramente mostramos que existem $\bar{v}$ e $\bar{j}$ tais que

$$
f(\bar{v}, \bar{j})=0 .
$$


Fixando $K=n^{2} \bar{N}+2 N$ e tomando a sequência $\gamma_{k}=1 / k, k \in \mathbb{Z}_{+}$. Temos de (3.10) que existem $v_{k} \in \mathbb{R}^{n}$ e $j_{k} \in \mathcal{S}$ tais que $f\left(v_{k}, j_{k}\right)<\gamma_{k}\left\|v_{k}\right\|^{2}$ para cada $k \geq 0$. Como o conjunto

$$
W=\left\{(u, j), u \in \mathbb{R}^{n}, j \in \mathcal{S}:\|u\|=1\right\}
$$

é compacto, comcluímos que a sequência $\left(v_{k} /\left\|v_{k}\right\|, j_{k}\right) \in W$ possui ao menos um ponto de acumulação $(\bar{v}, \bar{j}) \in W$. Tomando uma subsequência $\left(v_{k_{\ell}} /\left\|v_{k_{\ell}}\right\|, \bar{j}\right)$ convergente para o ponto $(\bar{v}, \bar{j})$, segue da continuidade de $f$ em seu primeiro argumento $v$ que

$$
f(\bar{v}, \bar{j})=f\left(\lim _{\ell \rightarrow \infty} v_{k_{\ell}} /\left\|v_{k_{\ell}}\right\|, \bar{j}\right) \leq \lim _{\ell \rightarrow \infty} \gamma_{k_{\ell}}=0
$$

levando a igualdade em (3.12).

Agora, da definição em (3.11), temos

$$
\bar{v}^{\prime} \mathcal{E}\left\{\sum_{t=2 N}^{n^{2} \bar{N}+2 N-1} \phi_{t, \theta(t)} \mid \theta(0)=\bar{j}\right\} \bar{v} \leq \bar{v}^{\prime} \mathcal{E}\left\{\sum_{t=0}^{n^{2} \bar{N}+2 N-1} \phi_{t, \theta(t)} \mid \theta(0)=\bar{j}\right\} \bar{v}=0
$$

Então

$$
\bar{v}^{\prime}\left(\sum_{t=2 N}^{n^{2} \bar{N}+2 N-1} \phi_{t, \theta(t)}\right) \bar{v}=0
$$

quase certamente quando $\theta(0)=\bar{j}$, isto é, para qualquer sequência $j_{0}=\bar{j}, j_{1}, j_{2}, \ldots$, que satisfaça

$$
\mathcal{P}\left(\theta(0)=j_{0}=\bar{j}, \theta(1)=j_{1}, \ldots, \theta\left(\left(n^{2} \bar{N}+2 N-1\right)\right)=j_{n^{2} \bar{N}+2 N-1}\right)>0 .
$$

Em particular, tomando $\ell$ tal que $\mathcal{S}_{\ell}$ seja alcançável a partir de $\bar{j}$ e considerando o conjunto de sequências da forma (3.14) para as quais $j_{N} \in \mathcal{S}_{\ell}$. Como o espaço de estados da cadeia de Markov é finito, este conjunto de sequências é finito. Denotamos este conjunto por

$$
\mathcal{M}=\left\{\left(m_{1}\right),\left(m_{2}\right), \ldots\left(m_{\varsigma}\right)\right\}, \text { onde }\left(m_{\ell}\right)=\left(j_{0}^{\left(m_{\ell}\right)}, j_{1}^{\left(m_{\ell}\right)} \ldots, j_{n^{2} N+2 N-1}^{\left(m_{\ell}\right)}\right)
$$

$\operatorname{com} j_{0}^{\left(m_{\ell}\right)}=\bar{j}$ e $j_{N}^{\left(m_{\ell}\right)} \in \mathcal{S}_{\ell}$. 
Para cada $\left(m_{\ell}\right) \in \mathcal{M}$ definimos

$$
\Gamma_{\ell}=\sum_{t=2 N}^{n^{2} \bar{N}+2 N-2} p_{j_{t}^{\left(m_{\ell}\right)} j_{t+1}^{\left(m_{\ell}\right)} \ldots p_{j_{n^{2}\left(m_{\ell}\right)}^{\left(m_{N}\right)-2} j_{n}^{2} \frac{\ell}{N}+2 N-1}^{\left(m_{\ell}\right)}} \phi_{t, j_{t}} 1_{\left\{j_{t} \in \mathcal{S}_{\ell}\right\}} .
$$

De (3.13), (3.14) e nas condições da definição acima, avaliamos $\bar{v}^{\prime} \Gamma_{\ell} \bar{v}=0$. Note que, como $j_{N} \in \mathcal{S}_{\ell}$, então $j_{t}, t \geq 2 N$ pertence a (e pode ser qualquer membro de) $\mathcal{S}_{\ell}$. Combinando isto com o fato de que os termos em (3.16) são os mesmos que aparecem na definição (3.5) com termos expandidos, ou seja,

$$
\sum_{\ell=1}^{\varsigma} \Gamma_{\ell}=\sum_{i \in \mathcal{S}_{\ell}} \mathcal{A}_{\ell, i}\left(\sum_{t=0}^{n^{2} \bar{N}-2} \mathcal{A}_{\ell}^{t}\left(M_{\ell}\right)\right)+M_{\ell, i}
$$

obtemos

$$
\begin{aligned}
0 & =\bar{v}^{\prime}\left(\mathcal{A}_{\ell, i}\left(\sum_{t=0}^{n^{2} \bar{N}-2} \mathcal{A}_{\ell}^{t}\left(M_{\ell}\right)\right)+M_{\ell, i}\right) \bar{v} \\
& =\bar{v}^{\prime}\left(\mathcal{A}_{\ell, i}\left(R_{\ell}\left(n^{2} \bar{N}-1\right)\right)+M_{\ell, i}\right) \bar{v} \\
& =\bar{v}^{\prime}\left(R_{\ell, i}\left(n^{2} \bar{N}\right)\right) \bar{v},
\end{aligned}
$$

para $i \in \mathcal{S}_{\ell}$. Isto e o Lema 3.1 implicam que $\operatorname{posto}\left(R_{\ell, i}\left(n^{2} N_{\ell}\right)\right)<n$.

(Necessidade) Assuma que exista $1 \leq \ell \leq r$, tal que posto $\left(\mathcal{R}_{\ell, i}\right)<n$, para algum $i \in \mathcal{S}_{\ell}$. Logo existe um vetor não trivial $v \in \mathbb{R}^{n}$ tal que $v \in \mathcal{K} \operatorname{er}\left\{\mathcal{R}_{\ell, i} \mathcal{R}_{\ell, i}^{\prime}\right\}$, levando a $v^{\prime}\left(\mathcal{R}_{\ell, i} \mathcal{R}_{\ell, i}^{\prime}\right) v=0$, e como consequência

$$
v^{\prime}\left(\sum_{t=1}^{n^{2} N_{\ell}} R_{\ell, i}(t) R_{\ell, i}^{\prime}(t)\right) v=0
$$

Isto e o Lema 3.1 implicam que

$$
v^{\prime}\left(R_{\ell, i}(t) R_{\ell, i}^{\prime}(t)\right) v=0, t=1,2, \ldots, n^{2} N_{\ell}
$$

logo do Lema 3.1, para cada $k \geq n^{2} N_{\ell}$, existe um vetor não trivial $v_{k} \in \mathbb{R}^{n}$, tais que $v_{k} \in$ $\mathcal{K} \operatorname{er}\left\{R_{\ell, i}(k)\right\}$. Similarmente a prova de suficiência, consideramos o conjunto de sequências 
$\mathcal{M}=\left\{\left(m_{1}\right),\left(m_{2}\right), \ldots, m_{\varphi}\right\}$. Para $\theta(0)=\bar{j} \in \mathcal{S}_{\ell}$, e para cada $\left(m_{\ell}\right) \in \mathcal{M}$ definimos

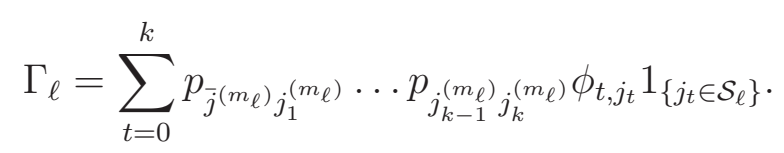

Pelo Corolário 3.1, $v_{k} \in \mathcal{K} e r\left\{R_{\ell, j}(k)\right\}$ para todo $j \in \mathcal{S}_{\ell}$, então

$$
\begin{aligned}
0 & =v_{k+1}^{\prime} \sum_{i \in \mathcal{S}_{\ell}}\left(R_{\ell, i}(k+1)\right) v_{k+1} \\
& =v_{k+1}^{\prime} \sum_{i \in \mathcal{S}_{\ell}}\left(\mathcal{A}_{\ell, i}(R(k))+M_{\ell, i}\right) v_{k+1}=v_{k+1}^{\prime} \sum_{\ell=1}^{\varphi} \Gamma_{\ell} v_{k+1}
\end{aligned}
$$

pode-se verificar a última igualdade expandindo-se $R_{\ell, i}$ definida em (3.5)-(3.6) e comparando os termos que aparecem na definição de $\Gamma$.

Logo, (3.17) fornece

$$
v_{k+1}^{\prime} \Gamma_{\ell} v_{k+1}=0,\left(m_{\ell}\right) \in \mathcal{M}
$$

levando a

$$
v_{k+1}^{\prime}\left(\mathcal{E}\left\{\sum_{t=0}^{k} \phi_{t, j_{t}} 1_{\left\{j_{t} \in \mathcal{S}_{\ell}\right\}} \mid \theta(0)=\bar{j}\right\}\right) v_{k+1}=0, \quad \bar{j} \in \mathcal{S}_{\ell} .
$$

Isto contradiz a M-alcançabilidade, concluindo a prova.

O resultado abaixo é o primeiro resultado de positividade apresentado e dele decorrem, direta ou indiretamente, todos os resultados de positividade abordados no decorrer do trabalho. Uma versão preliminar do Corolário 3.2 pode ser encontrada em [39], [40].

Corolário 3.2 Se $(A, B, P)$ é M-alcançável, então para cada $1 \leq \ell \leq r$ existe $\gamma_{R, \ell} \in \mathbb{R}_{+}$ tal que

$$
R_{\ell, i}(k) \geq \gamma_{R, \ell} I, i \in \mathcal{S}_{\ell}, k \geq n^{2} N_{\ell}
$$

Demonstração: Para $k \geq n^{2} N_{\ell}$, temos

$$
\begin{aligned}
R_{\ell}(k+1) & =\sum_{t=0}^{n^{2} N_{\ell}-1} \mathcal{A}_{\ell}^{t}\left(M_{\ell}\right)+\sum_{t=n^{2} N_{\ell}}^{k} \mathcal{A}_{\ell}^{t}\left(M_{\ell}\right) \\
& =R_{\ell}\left(n^{2} N_{\ell}\right)+\sum_{t=n^{2} N_{\ell}}^{k} \mathcal{A}_{\ell}^{t}\left(M_{\ell}\right) \geq R_{\ell}\left(n^{2} N_{\ell}\right) \geq \gamma_{R, \ell} \mathbb{I}
\end{aligned}
$$

onde a última desigualdade segue do Teorema 1. 


\subsubsection{Exemplos}

Os exemplos adiante ilustram o teste para M-alcançabilidade fornecido pelo teorema do posto completo.

Exemplo 3.1 Considere o sistema $\Phi_{M}$ em (2.4) com

$$
\begin{gathered}
A_{1}=\left[\begin{array}{ccc}
0 & 1,9 & 1 \\
1 & 0 & 0 \\
1 & 0 & 1
\end{array}\right], A_{2}=\left[\begin{array}{ccc}
0 & 0 & 1 \\
0 & 0 & 1,2 \\
1 & 0 & 1,3
\end{array}\right], B_{1}=\left[\begin{array}{ccc}
1 & 0 & 0
\end{array}\right]^{\prime}, B_{2}=\left[\begin{array}{lll}
1 & 0 & 1
\end{array}\right]^{\prime}, \\
C_{1}=C_{2}=D_{1}=D_{2}=I, P=\left[\begin{array}{ll}
0,1 & 0,9 \\
0,1 & 0,9
\end{array}\right] .
\end{gathered}
$$

$D e(3.7)$

$$
\begin{aligned}
& \mathcal{R}_{1,1}=R_{1,1}(18)=1,0 e+028 *\left[\begin{array}{ccc}
2,4 & 1,4 & 3,1 \\
1,4 & 0,8 & 1,8 \\
3,1 & 1,7 & 3,8
\end{array}\right], \text { posto }\left(\mathcal{R}_{1,1}\right)=3 \\
& \mathcal{R}_{1,2}=R_{1,2}(18)=1,0 e+028 *\left[\begin{array}{ccc}
2,4 & 1,4 & 3,1 \\
1,4 & 0,8 & 1,7 \\
3,1 & 1,7 & 3,8
\end{array}\right], \text { posto }\left(\mathcal{R}_{1,2}\right)=3
\end{aligned}
$$

portanto pelo Teorema do posto completo, o sistema $(A, B, P)$ é M-alcançável.

Exemplo 3.2 Considere o Exemplo $3.1 \mathrm{com}$

$$
\begin{gathered}
A_{1}=\left[\begin{array}{ccc}
0 & 0,1 & 0 \\
0 & 1,1 & 0 \\
0 & 0 & 0,9
\end{array}\right], A_{2}=\left[\begin{array}{ccc}
0,9 & 0,4 & 0 \\
0 & 0,5 & 0 \\
0 & 0,5 & 0,8
\end{array}\right], B_{1}=\left[\begin{array}{lll}
1 & 0 & 0
\end{array}\right] \\
B_{2}=\left[\begin{array}{lll}
0,1 & 0 & 0
\end{array}\right]^{\prime}, P=\left[\begin{array}{ll}
0,9 & 0,1 \\
0,9 & 0,1
\end{array}\right]
\end{gathered}
$$


De (3.7)

$$
\begin{aligned}
& \mathcal{R}_{1,1}=R_{1,1}(18)=\left[\begin{array}{ccc}
26,71 & 0 & 0 \\
0 & 0 & 0 \\
0 & 0 & 0
\end{array}\right], \operatorname{posto}\left(\mathcal{R}_{1,1}\right)=1 \\
& \mathcal{R}_{1,2}=R_{1,2}(18)=\left[\begin{array}{ccc}
26,71 & 0 & 0 \\
0 & 0 & 0 \\
0 & 0 & 0
\end{array}\right], \operatorname{posto}\left(\mathcal{R}_{1,2}\right)=1,
\end{aligned}
$$

portanto pelo Teorema do posto completo, o sistema $(A, B, P)$ não é M-alcançável. 


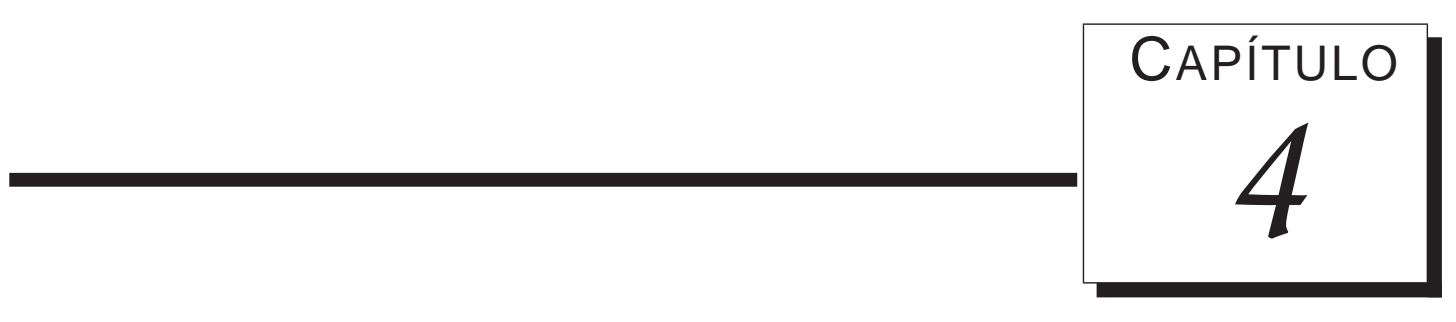

\section{Positividade do segundo momento}

Neste Capítulo, mostramos que M-alcançabilidade é uma condição necessária e suficiente para que o segundo momento de $x(k), \mathcal{E}\left\{x(k) x(k)^{\prime}\right\}$, seja limitado inferiormente por $\beta I$, para algum escalar positivo $\beta$, para instantes de tempo $k$ suficientemente grande, como apresentado na Proposição 4.1. Isso assegura que M-alcançabilidade é uma condição suficiente para a positividade da covariância do erro de estimação $\mathcal{E}\left\{Y^{k}(D, B, \Psi)\right\}$, veja o Teorema 2.

\subsection{Resultados auxiliares e ordenação de matrizes}

Embora as matrizes de M-alcançabilidade definidas em (3.7) não dependam dos valores das distribuições $\pi_{i}(k)$, estão relacionadas ao segundo momento do estado $x(k)$. A fim de mostrar que M-alcançabilidade é condição para positividade, definimos o conjunto de matrizes $S_{i}(k)$ que pode ser comparado com ambos $R_{i}(k)$ e $x(k)$. Seguindo esta mesma linha, definimos a seguir, certas matrizes $T_{i}(k), \bar{S}_{i}(k)$ e reunimos alguns resultados essenciais na prova da Proposição 4.1 e do Teorema 2. 


\subsubsection{Matrizes de M-alcançabilidade e segundo momento}

Definimos o operador $\mathcal{H}_{V}: \mathcal{N}^{N, n 0} \rightarrow \mathcal{N}^{N, r 0}$, de maneira que para $U \in \mathcal{N}^{N, r 0}$ e $V \in$ $\mathcal{N}^{N, r, n}$,

$$
\mathcal{H}_{V, i}(U)=\sum_{j=1}^{N} p_{j i} V_{j} U_{j} V_{j}^{\prime}, \quad i=1, \ldots, N
$$

Considere as matrizes $S_{i}(k) \in \mathcal{M}^{n 0}, i \in \mathcal{S}$, definidas como

$$
\begin{aligned}
S_{i}(k+1) & =\mathcal{H}_{A, i}(S(k))+\sum_{j=1}^{N} \pi_{j}(k) p_{j i} B_{j} B_{j}^{\prime}, \quad k \geq 0, \\
S_{i}(0) & =\Psi \pi_{i}(0) .
\end{aligned}
$$

$S_{i}(k)$ e $x(k)$ são relacionados como segue.

Lema 4.1 As seguintes afirmações valem:

1. $S_{i}(k)=\mathcal{E}\left\{x(k) x^{\prime}(k) 1_{\{\theta(k)=i\}}\right\}, \quad i \in \mathcal{S}, \quad k \geq 0$,

2. $\mathcal{E}\left\{X^{k}(B, \Psi) \mid \theta(k)=i\right\}=S_{i}(k) \mathcal{P}(\theta(k)=i)$.

Demonstração: A primeira afirmação segue diretamente de [23, Proposição 3.1]. A segunda afirmação segue da Proposição 2.2 e da afirmação do item 1. acima,

$$
\begin{aligned}
\mathcal{E}\left\{X^{k}(B, \Psi) \mid \theta(k)=i\right\} & =\mathcal{E}\left\{\mathcal{E}\left\{x(k) x^{\prime}(k) \mid \Theta_{k}\right\} \mid \theta(k)=i\right\} \\
& =\mathcal{E}\left\{x(k) x^{\prime}(k) 1_{\{\theta(k)=i\}}\right\} \mathcal{P}(\theta(k)=i)=S_{i}(k) \mathcal{P}(\theta(k)=i),
\end{aligned}
$$

completando a prova.

\subsubsection{Ordenação de matrizes}

Dada a relação entre os valores de $S_{i}(k)$ e $x(k)$ no Lema 4.1, definimos abaixo coleções de matrizes auxiliares, $T_{\ell}(k)$ e $\bar{S}_{\ell}(k)$ que, em função de propriedades de completude do posto de $R_{i}(k)$, fornecem um resultado de positividade para $S_{i}(k)$, Lema 4.6.

Introduzimos sequências de matrizes $T_{\ell}$ e $\bar{S}_{\ell}$, as quais são similares a $R_{\ell}$ - (3.5) e $S_{i}$ (4.2), respectivamente. 
Lembre-se que $K_{1}=K_{0}+n^{2} \bar{N}+N$. Para cada $1 \leq \ell \leq r$, define-se $T_{\ell, i}(k) \in \mathcal{M}^{n 0}$ como

$$
\begin{aligned}
T_{\ell, i}(k+1) & =\mathcal{A}_{\ell, i}\left(T_{\ell}(k)\right)+M_{\ell, i}, k \geq K_{1}, i \in \mathcal{S}_{\ell} \\
T_{\ell, i}\left(K_{1}\right) & =0, i \in \mathcal{S}_{\ell}
\end{aligned}
$$

e $\bar{S}_{\ell, i}(k) \in \mathcal{M}^{n 0}$ como

$$
\begin{aligned}
\bar{S}_{\ell, i}(k+1) & =\mathcal{A}_{\ell, i}\left(\bar{S}_{\ell}(k)\right)+M_{\pi, i}(k), i \in \mathcal{S}_{\ell} \\
\bar{S}_{\ell, i}(0) & =\Psi \pi_{i}(0)
\end{aligned}
$$

onde

$$
M_{\pi, i}(k)=\sum_{j=1}^{N} \pi_{j}(k) p_{j i} B_{j} B_{j}^{\prime} 1_{\left\{j \in \mathcal{S}_{\ell}\right\}}, i \in \mathcal{S}_{\ell} .
$$

Observe que $T_{\ell}$ é uma translação no tempo de $R_{\ell} \cdot \bar{S}_{\ell}$ é uma "versão local" de $S_{i}$, coincidindo $\operatorname{com} S_{i}$ quando a distribuição inicial $\pi_{i}(0)$ é igual a zero para qualquer estado $\theta(0)$ fora de $\mathcal{S}_{\ell}$

A seguir, mostramos que as matrizes $S_{i}(k), \bar{S}_{\ell, i}(k)$ e $T_{\ell, i}(k)$ podem ser ordenadas.

Lema 4.2 Considere as matrizes $S_{i}(k)$ em (4.2) e $\bar{S}_{\ell, i}(k)$ em (4.4), então para cada $1 \leq \ell \leq$ $r$, e $i \in \mathcal{S}_{\ell}$, tem-se $S_{i}(k) \geq \bar{S}_{\ell, i}(k), \forall k \geq 0$.

Demonstração: Denotando $N_{\pi, i}(k)=\sum_{j=1}^{N} \pi_{j}(k) p_{j i} B_{j} B_{j}^{\prime}$, tem-se $N_{\pi, i} \geq M_{\pi, i}, i \in \mathcal{S}_{\ell}$. Temos que

$$
\begin{aligned}
S_{i}(k+1) & =\mathcal{H}_{A, i}\left[\mathcal{H}_{A}^{k}(\Psi \pi(0))+\sum_{t=0}^{k-1} \mathcal{H}_{A}^{t}\left(N_{\pi}(k-t)\right)\right]+N_{\pi, i}(k) \\
& \geq \mathcal{A}_{\ell, i}\left[\mathcal{A}_{\ell}^{k}(\Psi \pi(0))+\sum_{t=0}^{k-1} \mathcal{A}_{\ell}^{t}\left(M_{\pi}(k-t)\right)\right]+M_{\pi, i}(k) \\
& =\bar{S}_{\ell, i}(k+1), \quad \forall k \geq 0, i \in \mathcal{S}_{\ell},
\end{aligned}
$$

e a prova está completa.

Lema 4.3 Assuma que $\ell$ seja tal que $\mathcal{S}_{\ell} \in R_{\pi}$. Então existe $\iota \in \mathbb{R}_{+}$, tal que $\bar{S}_{\ell, i}(k) \geq$ $\iota \mathcal{P}\left(\theta(N) \in \mathcal{S}_{\ell}\right) T_{\ell, i}(k), \forall i \in \mathcal{S}_{\ell}, k \geq K_{1}$. 
Demonstração: Segue da Proposição 2.1 que para $\ell$ satisfazendo $\mathcal{S}_{\ell} \in R_{\pi}$ existe $0<\iota<1$ tal que $\pi_{j}(k) \geq \iota \mathcal{P}\left(\theta(N) \in \mathcal{S}_{\ell}\right), j \in \mathcal{S}_{\ell}, k \geq K_{0}$, portanto de (4.3) e (4.4) tem-se

$$
\begin{aligned}
\bar{S}_{\ell, i}(k+1)=\mathcal{A}_{\ell, i}\left[\mathcal{A}_{\ell}^{k}(\Psi \pi(0))+\sum_{t=0}^{k-1} \mathcal{A}_{\ell}^{t}\left(M_{\pi}(k-t)\right)\right]+M_{\pi, i}(k) \\
\geq \mathcal{A}_{\ell, i}\left[\mathcal{A}_{\ell}^{k}(\Psi \pi(0))+\sum_{t=0}^{k-1} \mathcal{A}_{\ell}^{t}\left(M_{\pi}(k-t)\right)\right]+\iota \mathcal{P}\left(\theta(N) \in \mathcal{S}_{\ell}\right) M_{\ell, i} \\
\geq \mathcal{A}_{\ell, i}\left[\mathcal{A}_{\ell}^{k}(\Psi \pi(0))\right]+\mathcal{A}_{\ell, i}\left[\sum_{t=0}^{K_{0}-1} \mathcal{A}_{\ell}^{t}\left(M_{\pi}(k-t)\right)\right] \\
\quad+\iota \mathcal{P}\left(\theta(N) \in \mathcal{S}_{\ell}\right)\left[\mathcal{A}_{\ell, i}\left(\sum_{t=K_{0}}^{k-1} \mathcal{A}_{\ell}^{t}\left(M_{\ell}\right)\right)+M_{\ell, i}\right] \\
=\mathcal{A}_{\ell, i}\left[\mathcal{A}_{\ell}^{k}(\Psi \pi(0))\right]+\mathcal{A}_{\ell, i}\left[\sum_{t=0}^{K_{0}-1} \mathcal{A}_{\ell}^{t}\left(M_{\pi}(k-t)\right)\right]+\iota \mathcal{P}\left(\theta(N) \in \mathcal{S}_{\ell}\right) T_{\ell, i}(k+1) \\
\geq \iota \mathcal{P}\left(\theta(N) \in \mathcal{S}_{\ell}\right) T_{\ell, i}(k+1),
\end{aligned}
$$

concluindo a prova.

Lema 4.4 Se existirem $\gamma_{R}>0$ e $K \geq 0$, tais que $R_{\ell, i}(k) \geq \gamma_{R} I, \forall k \geq K$, então existem $\gamma_{T} \geq 0$ e $K_{T} \geq 0$, tais que $T_{\ell, i}(k) \geq \gamma_{T} I, k \geq K_{T}, i \in \mathcal{S}_{\ell}$.

Demonstração: O resultado a seguir segue diretamente das definições das matrizes $T_{i}(k) \mathrm{e}$ $R_{i}(k)$ (veja (3.5)), tomando $K_{T} \geq K+n^{2} \bar{N}$.

Lema 4.5 Considere o sistema em (2.4) e assuma $x_{0}$. Se existir um vetor não trivial $v \in$ $\mathcal{K}$ er $\left\{R_{\ell, i}(k)\right\}$, então $v \in \mathcal{K} \operatorname{er}\left\{\bar{S}_{\ell, i}(k)\right\}$, para todo $k \geq K$.

Demonstração: A prova segue do fato de que, quando $x_{0}=0$, a expressão que define $R_{\ell, i}(k)$ é análoga à de $\bar{S}_{\ell, i}(k), i \in \mathcal{S}_{\ell}$, se diferenciando somente por escalares positivos $\pi_{j}(k) \geq \iota \geq 0$.

\subsection{Positividade do processo $X(k)$}

Nesta seção, mostramos que M-alcançabilidade é uma condição necessária e suficiente para positividade de $\mathcal{E}\{X(k)\}$. A prova segue da relação entre $S_{i}(k)$ e $x(k)$, Lema 4.1, e das 
relações de ordenação apresentadas acima.

Lema 4.6 Valem as seguintes afirmações:

1. Se $(A, B, P)$ é M-alcançável, então existe $\beta \in \mathbb{R}_{+}$(independente de $\Psi$ ) tal que, para cada distribuição inicial $\pi=\pi(0), 1 \leq \ell \leq$ e e $i \in \mathcal{S}_{\ell} \cap R_{\pi}$,

$$
S_{i}(k) \geq \beta \mathcal{P}\left(\theta(N) \in \mathcal{S}_{\ell}\right) I \quad k \geq K_{1}
$$

2. Assuma que $(A, B, P)$ não seja M-alcançável e $\Psi=0$. Então existem uma distribuição inicial $\pi=\pi(0)$ e $i \in R_{\pi}$ tal que $S_{i}(k)$ é singular para algum $k \geq 0$.

\section{Demonstração:}

1. Do Corolário 3.2 temos que $R_{i}(k) \geq \gamma_{R} I, k \geq n^{2} \bar{N}$, portanto dos Lemas 4.2-4.4, temos para $i \in \mathcal{S}_{\ell} \cap R_{\pi}, k \geq K_{0}+n^{2} \bar{N}$,

$$
S_{i}(k) \geq \bar{S}_{\ell, i}(k) \geq \iota \mathcal{P}\left(\theta(N) \in \mathcal{S}_{\ell}\right) T_{\ell, i}(k) \geq \iota \mathcal{P}\left(\theta(N) \in \mathcal{S}_{\ell}\right) \gamma_{T} I
$$

2. Para um sistema não alcançável, de acordo com o Teorema 1, existe $m$ tal que $\operatorname{posto}\left(\mathcal{R}_{m, i}\right)<n$, para algum $i \in \mathcal{S}_{m}$. Considere $\pi=\pi(0)$ de forma que $R_{\pi}=\mathcal{S}_{m}$. Obtemos do Lema 3.1 que posto $\left(R_{m, i}(k)\right)<n, k \geq 0$, e o Corolário 3.1 fornece $\operatorname{posto}\left(R_{m, j}(k)\right)<n, k \geq 0, \forall j \in \mathcal{S}_{m}$. Agora, escolhendo $x(0)=0$, obtemos do Lema 4.5 que

$$
\operatorname{posto}\left(\bar{S}_{m, j}(k)\right)<n, \quad \forall k \geq 0, \forall j \in \mathcal{S}_{m}
$$

Por outro lado, para $\pi$ como acima e para cada $k \geq 0$ temos que $\pi_{j}(k) p_{j \ell}=0$ sempre que $j \notin \mathcal{S}_{m}$ ou $\ell \notin \mathcal{S}_{m}$, obtemos de (4.2) e (4.4) que $S_{\ell}(k)=\bar{S}_{m, \ell}(k), \ell \in \mathcal{S}_{m}$ e $S_{\ell}(k)=0, \ell \notin \mathcal{S}_{m}$. Isto e (4.5) implicam que, para cada $j \in \mathcal{S}$, existe $v \in \mathbb{R}^{n}$ tal que $v \in \mathcal{K} \operatorname{er}\left\{S_{j}(k)\right\}, k \geq 0$, e a afirmação do lema segue escolhendo-se um valor particular de $j \in R_{\pi}$.

A seguinte proposição relaciona o conceito de M-alcançabilidade com a covariância do estado $X^{k}(B, \Psi)$. Um resultado similar pode ser encontrado em [39, Lemma 3] sob a hipótese de controlabilidade fraca e da restrição de ergodicidade sobre a cadeia de Markov. 
Proposição 4.1 Considere o conjunto de matrizes dado por (2.10). $\quad(A, B, P)$ é M-alcançável se e somente se, para toda distribuição inicial $\pi=\pi(0)$ e $i \in R_{\pi}$,

$$
\mathcal{E}\left\{X^{k}(B, 0) \mid \theta(k)=i\right\}>\beta \kappa I, \quad k \geq K_{1},
$$

com $\beta$ e $\kappa$ definidos respectivamente como no Lema 4.6 e Proposição 2.1.

Demonstração: (Necessidade) Para todo $\pi, i \in R_{\pi}$ e $k \geq N$, temos de propriedades básicas da cadeia de Markov que $\mathcal{P}(\theta(k)=i)>0$. Por esta propriedade, do fato de que $K_{1} \geq N$, pelos Lemas 4.1(2) - 4.6(1) para sistemas alcansáveis, podemos avaliar para $k \geq K_{1}$,

$$
\begin{aligned}
\mathcal{E}\left\{X^{k}(B, 0) \mid \theta(k)=i\right\} & =S_{i}(k) \mathcal{P}(\theta(k)=i) \geq \beta \mathcal{P}\left(\theta(N) \in \mathcal{S}_{\ell}\right) \mathcal{P}(\theta(k)=i) I \\
& \geq \beta \kappa I,
\end{aligned}
$$

onde $\ell$ é tal que $i \in \mathcal{S}_{\ell}$ e a última desigualdade segue da Proposição 2.1.

(Suficiência) Provamos por contradição. Suponha que $(A, B, P)$ não seja M-alcançável, então pelo Teorema 1, existe $m$ tal que $\operatorname{posto}\left(\mathcal{R}_{m, i}\right)<n$, para algum $i \in S_{m}$. Tomando $\pi(0)$ tal que $S_{m}=R_{\pi}$ e $x(0)=0$ (tal que $\Psi=0$ ), obtemos do Lema 4.6 e Lema 4.1 que existe um vetor não trivial $v \in \mathbb{R}^{n}$, satisfazendo

$$
0=v^{\prime}\left(\sum_{j=1}^{N} S_{j}(k)\right) v \geq v^{\prime} S_{i}(k) v=\mathcal{P}(\theta(k)=i) v^{\prime}\left(\mathcal{E}\left\{X^{k}(B, 0) \mid \theta(k)=i\right\}\right) v .
$$

Como $i \in R_{\pi}$, tem-se $\mathcal{P}(\theta(k)=i)>0, k \geq K_{1}$, e as equações acima implicam

$$
v^{\prime}\left(\mathcal{E}\left\{X^{k}(B, 0) \mid \theta(k)=i\right\}\right) v=0 .
$$

O resultado da Proposição 4.1 é o primeiro passo para obtermos um limitante inferior para a covariância do erro em média $\mathcal{E}\{Y(k)\}$, o que será estudado no capítulo seguinte.

O corolário abaixo segue diretamente da Proposição 4.1 e da Propriedade 4. do valor esperado.

Corolário 4.1 $(A, B, P)$ é M-alcançável se e somente se,

$$
\mathcal{E}\left\{X^{k}(B, 0)\right\}>\beta \kappa^{-1} I, \quad k \geq K_{1}
$$


$\operatorname{com} \beta$ e $\kappa$ definidos respectivamente como no Lema 4.6 e Proposição 2.1.

$\mathrm{ex}_{3} .1$ 


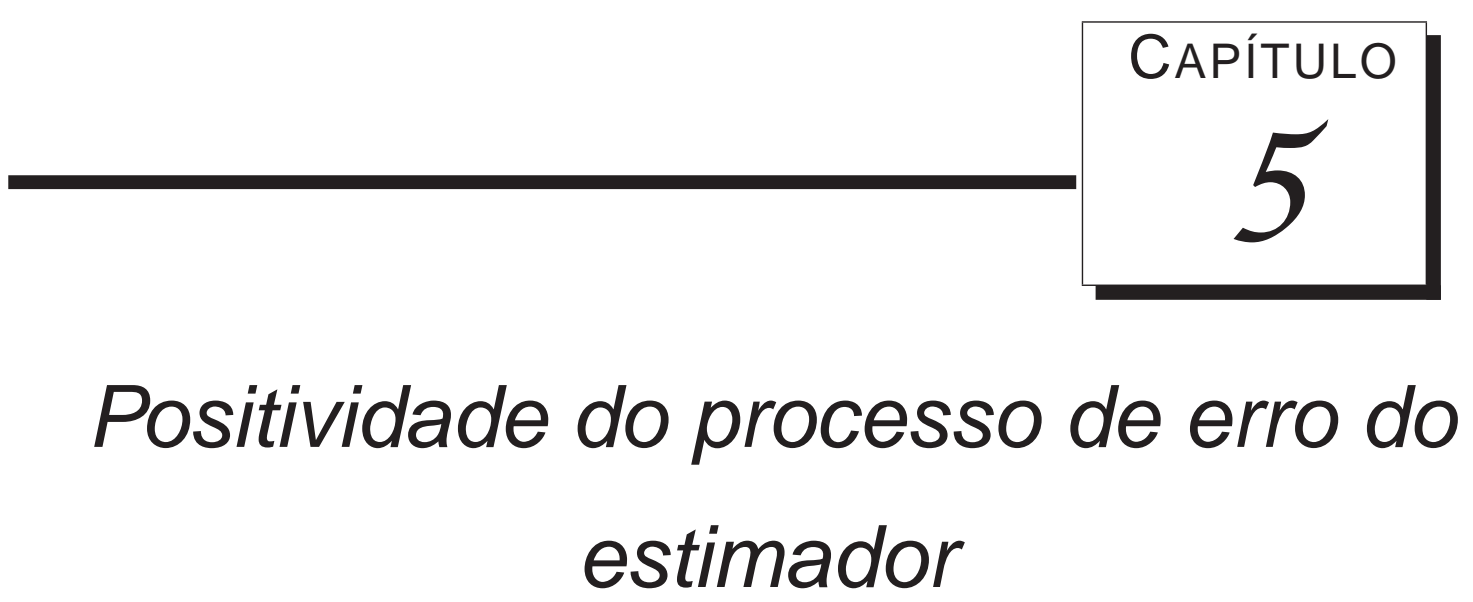

Nossa estratégia para obter um limitante inferior para $Y(k)$ (similar a limitação de $X(k)$ na Proposição 4.1) é essencialmente simples: mostra-se que $X(k)$ e $Y(k)$ estão relacionados de forma que " $Y$ pequeno" implica " $X$ pequeno". Uma interpretação é que um filtro linear não é capaz de eliminar ruídos em subespaços nos quais o ruído está presente no estado, o que é um fato bem conhecido para sistemas lineares variantes no tempo. Aqui, revisitamos este resultado, pois precisamos de informações mais precisas sobre as relações entre os valores de $Y(k)$ e $X(k)$. De fato, mostramos que: para qualquer $v \in \mathbb{R}^{n}$, tal que $v^{\prime} Y(k) v$ seja $O(\epsilon)$ implica que $v^{\prime} X(k) v$ também é $O\left(\epsilon^{1 / 2^{k}}\right)$. Onde $O(\epsilon)$ se refere a notação de Landau.

\subsection{Positividade do processo $\mathrm{Y}(\mathrm{k})$}

Iniciamos com uma generalização de [39, Corolário 3], onde se assume a condição restritiva de que $\pi_{i}(k) \geq \alpha, k \geq 0, i \in \mathcal{S}$ para algum $\alpha \in \mathbb{R}_{+}$.

Lema 5.1 Seja $K_{2}$ um inteiro positivo, $m=\left\{j_{0}, \ldots, j_{K_{2}}\right\}$ uma realização truncada da cadeia de Markov e $Y(k, m)$ a solução da equação da covariância do erro de estimação, (2.10). Existe $\zeta>0$ tal que para cada $0 \leq k \leq K_{2}-1, v \in \mathbb{R}^{n}$ e $\epsilon>0$ satisfazendo 
$v^{\prime} Y(k+1, m) v<\epsilon\|v\|^{2}$ tem-se:

$$
v^{\prime} A_{j_{k}}^{\prime} Y(k, m) A_{j_{k}} v \leq \zeta^{2} \epsilon\|v\|^{2}
$$

Demonstração: A prova é trivial para $v=0$. Como a variável aleatória $Y(k)$ satisfaz a equação em (2.10), possuindo assim coeficientes limitados (independente do histórico da cadeia), existe um limitante superior para $Y(k)$ em intervalos de tempo fixos, ou seja, existe $\hat{Y}$ tal que $\|Y(k)\|<\hat{Y}$ q.c, $0 \leq k \leq K_{2}$. Lembrando que $Y(k)=Y^{k}(D, B, \Psi)$, avalia-se de (2.10) que

$$
v^{\prime} G(k, m) D_{j_{k}} D_{j_{k}}^{\prime} G^{\prime}(k, m) v \leq v^{\prime} Y(k+1, m) v<\epsilon\|v\|^{2} .
$$

Denotando $\chi=\min _{i}\left\{\sigma_{1}\left(D_{i} D_{i}^{\prime}\right)\right\}$ temos $\chi v^{\prime} G(k, m) G^{\prime}(k, m) v<\epsilon\|v\|^{2}$, portanto

$$
\left\|G^{\prime}(k, m) v\right\|^{2}<\frac{\epsilon\|v\|^{2}}{\chi} .
$$

Por outro lado, de (2.10) tem-se

$$
v^{\prime}\left(A_{j_{k}}-G(k, m) C_{j_{k}}\right) Y(k, m)\left(A_{j_{k}}-G(k, m) C_{j_{k}}\right)^{\prime} v<\epsilon\|v\|^{2},
$$

que pode ser escrito como

$$
\begin{aligned}
v^{\prime}\left(A_{j_{k}} Y(k, m) A_{j_{k}}^{\prime}-A_{j_{k}} Y(k, m) C_{j_{k}}^{\prime}(k)\right. & G^{\prime}(k, m)-G(k, m) C_{j_{k}} Y(k, m) A_{j_{k}}^{\prime} \\
& \left.+G(k, m) C_{j_{k}} Y(k, m) C_{j_{k}}^{\prime} G^{\prime}(k, m)\right) v<\epsilon\|v\|^{2},
\end{aligned}
$$

ou

$$
\begin{aligned}
\left\|Y^{\frac{1}{2}}(k, m) A_{j_{k}}^{\prime} v\right\|^{2} & +\left\|Y^{\frac{1}{2}}(k, m) C_{j_{k}}^{\prime} G^{\prime}(k, m) v\right\| \\
& <v^{\prime} A_{j_{k}} Y(k, m) C_{j_{k}}^{\prime} G^{\prime}(k, m) v+v^{\prime} G(k, m) C_{j_{k}} Y(k, m) A_{j_{k}}^{\prime} v+\epsilon\|v\|^{2} \\
& \leq 2\left\|v^{\prime} G(k, m) C_{j_{k}} Y(k, m) A_{j_{k}}^{\prime} v\right\|+\epsilon\|v\|^{2} \\
& \leq 2 \hat{Y}\left\|G^{\prime}(k, m) v\right\|\left\|C_{j_{k}}\right\|\left\|Y^{\frac{1}{2}}(k, m) A_{j_{k}}^{\prime} v\right\|+\epsilon\|v\|^{2},
\end{aligned}
$$

onde utilizamos a desigualdade de Cauchy-Schwarz e a consistência da norma nas desigualdades acima. Utilizando (5.1), tem-se

$$
\left\|Y^{\frac{1}{2}}(k, m) A_{j_{k}}^{\prime} v\right\|^{2} \leq 2 \sqrt{\epsilon / \chi}\|v\|\left(\hat{Y} \max _{i}\left(\left\|C_{i}\right\|\right)\right)\left\|Y^{\frac{1}{2}}(k, m) A_{j_{k}}^{\prime} v\right\|+\epsilon\|v\|^{2}
$$


Seja $\alpha$ a única raiz real e positiva de $\alpha^{2}+2 \sqrt{1 / \chi}\left(\hat{Y} \max _{i}\left(\left\|C_{i}\right\|\right)\right) \alpha-1$, de forma que $(1 / \alpha)-\alpha=2 \sqrt{1 / \chi}\left(\hat{Y} \max _{i}\left(\left\|C_{i}\right\|\right)\right)$, o que permite escrever

$$
\begin{aligned}
& \left(\left\|Y^{\frac{1}{2}}(k, m) A_{j_{k}}^{\prime} v\right\|+\alpha \sqrt{\epsilon}\|v\|\right)\left(\left\|Y^{\frac{1}{2}}(k, m) A_{j_{k}}^{\prime} v\right\|-\frac{\sqrt{\epsilon}\|v\|}{\alpha}\right) \\
& =\left\|Y^{\frac{1}{2}}(k, m) A_{j_{k}}^{\prime} v\right\|^{2}-2 \sqrt{1 / \chi}\left(\hat{Y} \max _{i}\left(\left\|C_{i}\right\|\right)\right)(\sqrt{\epsilon}\|v\|)\left\|Y^{\frac{1}{2}}(k, m) A_{j_{k}}^{\prime} v\right\|-\epsilon\|v\|^{2} \\
& \leq 0 .
\end{aligned}
$$

onde a desigualdade segue imediatamente de (5.2). Como $\alpha>0$ e $v$ é não trivial, multiplicamos a desigualdade acima pelo inverso de $\left\|Y^{\frac{1}{2}}(k, m) A_{j_{k}}^{\prime} v\right\|+\alpha \sqrt{\epsilon}\|v\|$ para obter

$$
\left\|Y^{\frac{1}{2}}(k, m) A_{j_{k}}^{\prime} v\right\|-\frac{\sqrt{\epsilon}\|v\|}{\alpha} \leq 0
$$

e o resultado segue $\operatorname{com} \zeta=\alpha^{-1}$.

Lema 5.2 Considere $0 \leq k \leq K_{2}$ e seja $\left\{j_{0}^{(m)}, \ldots, j_{K_{2}}^{(m)}\right\}$ qualquer realização truncada fixa da cadeia de Markov e $Y(k, m)$ correspondendo a equação (2.10). Então vale o seguinte, se existem $v \in \mathbb{R}^{n}$ e $\epsilon>0$ tais que $v^{\prime} Y(k, m) v<\epsilon\|v\|^{2}$, então existe $h_{k} \in O\left(\epsilon^{\frac{1}{2^{k}}}\right)$ tal que $v^{\prime} X^{k}(B, \Psi, m) v<h_{k}(\epsilon)\|v\|^{2}, 0 \leq k \leq K_{1}$.

Demonstração: Inicialmente, é simples verificar que existe $\mathcal{X}>0$ tal que $\left\|X^{k}(B, \Psi, m)\right\| \leq \mathcal{X}, \forall 0 \leq k \leq K_{1}$. A prova do resultado é feita por indução. Para $k=0$, temos $Y(0, m)=\Psi=X^{0}(B, \Psi, m)$, e o resultado é imediato com $h_{0}(\epsilon)=\epsilon$. Suponha por indução que o resultado do lema seja válido para $k>0$. Considere $v \in \mathbb{R}^{n}$ satisfazendo $v^{\prime} Y(k+1, m) v<\epsilon\|v\|^{2}$, temos do Lema 5.1 que existe $\zeta>0$ tal que

$$
v^{\prime} A_{j_{k}} Y(k, m) A_{j_{k}}^{\prime} v \leq \zeta^{2} \epsilon\|v\|^{2}
$$

Agora, consideramos dois casos possíveis:

1. Se $\left\|A_{j_{k}} v\right\|^{2} \geq \sqrt{\epsilon}\|v\|^{2}$, então

$$
v^{\prime} A_{j_{k}} Y(k, m) A_{j_{k}}^{\prime} v \leq \zeta^{2} \epsilon\|v\|^{2} \leq \zeta^{2} \sqrt{\epsilon}\left\|A_{j_{k}} v\right\|^{2}
$$


o que pela hipótese de indução leva a

$$
v^{\prime} A_{j_{k}} X^{k}(B, \Psi, m) A_{j_{k}}^{\prime} v<h_{k}\left(\zeta^{2} \sqrt{\epsilon}\right)\left\|A_{j_{k}} v\right\|^{2} \leq h_{k}\left(\zeta^{2} \sqrt{\epsilon}\right)\left\|A_{j_{k}}\right\|^{2}\|v\|^{2} .
$$

2. Se $\left\|A_{j_{k}} v\right\|^{2} \leq \sqrt{\epsilon}\|v\|^{2}$, então

$$
\begin{aligned}
v^{\prime} A_{j_{k}} X^{k}(B, \Psi, m) A_{j_{k}}^{\prime} v & \leq\left\|A_{j_{k}}^{\prime} v\right\|^{2}\left\|X^{k}(B, \Psi, m)\right\| \\
& \leq \sqrt{\epsilon}\|v\|^{2}\left\|X^{k}(B, \Psi, m)\right\| \leq \sqrt{\epsilon} \mathcal{X}\|v\|^{2} .
\end{aligned}
$$

Combinando as desigualdades acima tem-se

$$
v^{\prime} A_{j_{k}} X^{k}(B, \Psi, m) A_{j_{k}}^{\prime} v \leq \max \left(\mathcal{X},\left\|A_{1}\right\|^{2},\left\|A_{2}\right\|^{2}, \ldots,\left\|A_{N}\right\|^{2}\right) h_{k}\left(\zeta^{2} \sqrt{\epsilon}+\sqrt{\epsilon}\right)\|v\|^{2},
$$

o que implica

$$
v^{\prime} A_{j_{k}} X^{k}(B, \Psi, m) A_{j_{k}}^{\prime} v \leq \tilde{h}_{k}(\epsilon)\|v\|^{2}
$$

onde $\tilde{h}_{k}$ é $O\left((\sqrt{\epsilon})^{\frac{1}{2^{k}}}\right)=O\left(\epsilon^{\frac{1}{2 k+1}}\right)$.

Agora temos da hipótese do Lema e de (2.10) que $v^{\prime} B_{j_{k}} B_{j_{k}}^{\prime} v<\epsilon\|v\|^{2}$. Substituindo isto e (5.3) em (2.5) obtemos

$$
v^{\prime} X^{k+1}(B, \Psi, m) v=v^{\prime} A_{j_{k}} X^{k}(B, \Psi, m) A_{j_{k}}^{\prime} v+v^{\prime} B_{j_{k}} B_{j_{k}}^{\prime} v \leq\left(\tilde{h}_{k}(\epsilon)+\epsilon\right)\|v\|^{2},
$$

portanto, o resultado vale para $k+1$, completando a prova.

A partir de agora queremos modificar um valor esperado condicional sem mudar a distribuição da cadeia de Markov, veja a Observação 3. A relação entre as magnitudes de $X$ e $Y$ obtida nos lemas acima é essencial na extensão da limitação inferior de $X$ para $Y$, como apresentamos a seguir.

Teorema 2 (Positividade do processo de erro) Assuma ( $A, B, P)$ M-alcançável e considere o processo de erro $\{Y(k)\}$. Existe $\lambda \in \mathbb{R}_{+}$, tal que, para toda distribuição inicial $\pi_{0}$ e $K_{1} \leq k \leq K_{2}$,

$$
\mathcal{E}\{Y(k) \mid \theta(k)=i, \theta(0)=j, Y(0)\}>\lambda I, \quad i, j \in R_{\pi}
$$


Demonstração: Denotamos o conjunto de distintas realizações de uma cadeia de Markov no intervalo de tempo $K_{1} \leq k \leq K_{2}$ por $\mathcal{M}=\left\{m_{1}, m_{2}, \ldots m_{\varepsilon}\right\}$, de forma que $m_{\ell}=\left(j_{K_{1}}^{\ell}, \ldots, j_{K_{2}}^{\ell}\right)$ seja uma realização. Temos um número finito $\varepsilon$ de (diferentes) realizações, uma vez que o espaço de estado da cadeia de Markov é finito. Seja $\mathcal{X} \in \mathbb{R}_{+}$tal que $\left\|X^{k}(B, \Psi, m)\right\| \leq \mathcal{X}, K_{1} \leq k \leq K_{2}$. Relacionado ao valor de $\mathcal{X}$, definimos $\mathcal{M}_{1} \subset \mathcal{M}$ por

$$
\mathcal{M}_{1}=\left\{m_{\ell} \in \mathcal{M}: \mathcal{P}\left(m_{\ell} \mid \theta(k), \theta(0), Y(0)\right) \leq \frac{\beta}{2 \kappa \mathcal{X} \varepsilon}\right\}
$$

onde $\beta, \kappa$ é como no Lema 4.1. Denota-se por $\mathcal{M}_{2}$ ao conjunto complementar de $\mathcal{M}_{1}$. Procedemos por contradição, supondo que para todo $\lambda>0$ existam $v \in \mathbb{R}^{n}$ e $K_{1} \leq k \leq K_{2}$ tais que $v^{\prime} \mathcal{E}\{Y(k) \mid \theta(k), \theta(0), Y(0)\} v \leq \lambda\|v\|^{2}$, podemos escrever

$$
v^{\prime} \mathcal{E}\{Y(k) \mid \theta(k), \theta(0), Y(0)\} v=\sum_{\ell=1}^{\varepsilon} \mathcal{P}\left(m_{\ell} \mid \theta(k), \theta(0), Y(0)\right) v^{\prime} Y\left(k, m_{\ell}\right) v \leq \lambda\|v\|^{2}
$$

o que leva a

$$
v^{\prime} Y\left(k, m_{\ell}\right) v \leq 2 \lambda\|v\|^{2} \kappa \mathcal{X} \varepsilon \beta^{-1}, m_{\ell} \in \mathcal{M}_{2}
$$

e do Lema 5.2 obtém-se

$$
v^{\prime} X^{k}\left(B, \Psi, m_{\ell}\right) v \leq h_{k}\left(2 \lambda \kappa \mathcal{X} \varepsilon \beta^{-1}\right)\|v\|^{2}, m_{\ell} \in \mathcal{M}_{2},
$$

onde $h_{k}$ é $O\left((\cdot)^{\frac{1}{2^{k}}}\right)$. Portanto

$$
\begin{aligned}
v^{\prime} \mathcal{E}\left\{X^{k}(B, \Psi) \mid \theta(k), \theta(0), Y(0)\right\} v=\sum_{\ell=1}^{\varepsilon} \mathcal{P}\left(m_{\ell} \mid \theta(k), \theta(0), Y(0)\right) v^{\prime} X^{k}\left(B, \Psi, m_{\ell}\right) v \\
=\sum_{\ell \in \mathcal{M}_{1}} \mathcal{P}\left(m_{\ell} \mid \theta(k), \theta(0), Y(0)\right) v^{\prime} X^{k}\left(B, \Psi, m_{\ell}\right) v \\
\quad+\sum_{\ell \in \mathcal{M}_{2}} \mathcal{P}\left(m_{\ell} \mid \theta(k), \theta(0), Y(0)\right) v^{\prime} X^{k}\left(B, \Psi, m_{\ell}\right) v \\
\leq \sum_{\ell \in \mathcal{M}_{1}} \frac{\beta}{2 \kappa \mathcal{X} \varepsilon}\left\|X^{k}\left(B, \Psi, m_{\ell}\right)\right\|\|v\|^{2} \\
\quad+\sum_{\ell \in \mathcal{M}_{2}} \mathcal{P}\left(m_{\ell} \mid \theta(k), \theta(0), Y(0)\right) h_{k}\left(\frac{2 \lambda \kappa \mathcal{X} \varepsilon}{\beta}\right)\|v\|^{2}
\end{aligned}
$$




$$
\begin{aligned}
& =\frac{\beta}{2 \kappa \mathcal{X} \varepsilon}\|v\|^{2} \sum_{\ell \in \mathcal{M}_{1}}\left\|X^{k}\left(B, \Psi, m_{\ell}\right)\right\| \\
& \quad+h_{k}\left(\frac{2 \lambda \kappa \mathcal{X} \varepsilon}{\beta}\right)\|v\|^{2} \sum_{\ell \in \mathcal{M}_{2}} \mathcal{P}\left(m_{\ell} \mid \theta(k), \theta(0), Y(0)\right) \\
& \leq \frac{\beta}{2 \kappa \mathcal{X} \varepsilon}\|v\|^{2} \mathcal{X} \varepsilon+h_{k}\left(\frac{2 \lambda \kappa \mathcal{X} \varepsilon}{\beta}\right)\|v\|^{2} \\
& =\left(\frac{\beta}{2 \kappa}+h_{k}\left(\frac{2 \lambda \kappa \mathcal{X} \varepsilon}{\beta}\right)\right)\|v\|^{2} .
\end{aligned}
$$

Lembrando que $h_{k}$ é $O\left((\cdot)^{\frac{1}{2^{k}}}\right)$, basta tomar $\lambda>0$ suficientemente pequeno tal que $h_{k}\left(\frac{2 \lambda \kappa \mathcal{X} \varepsilon}{\beta}\right)<\frac{\beta}{2 \kappa}$, e a equação acima implica

$$
v^{\prime} \mathcal{E}\left\{X^{k}(B, \Psi) \mid \theta(k), \theta(0), Y(0)\right\} v \leq \beta \kappa^{-1}\|v\|^{2}
$$

Tomando o valor esperado condicional $\mathcal{E}\{\cdot \mid \theta(k)\}$, obtém-se

$$
v^{\prime} \mathcal{E}\left\{X^{k}(B, \Psi) \mid \theta(k)\right\} v \leq \beta \kappa^{-1}\|v\|^{2}
$$

de forma que $(A, B, P)$ não é M-alcançável de acordo com a Proposição 4.1.

A fim de abordar o caso em que $Y(0)=\Psi \geq 0$ é não trivial, note que

$$
\mathcal{E}\{Y(k) \mid \theta(k), \theta(0), Y(0)=\Psi\} \geq \mathcal{E}\{Y(k) \mid \theta(k), \theta(0), Y(0)=0\}>\lambda I,
$$

implicando no resultado seguinte.

Corolário 5.1 Assuma $(A, B, P)$ M-alcançável e considere a matriz de covariância do erro de estimação definida em (2.10). Então, existe $\lambda \in \mathbb{R}_{+}$, tal que, para toda distribuição inicial $\pi=\pi(0), i, j \in R_{\pi}, K_{1} \leq k \leq K_{2}$ e $M \in \mathcal{M}^{n 0}$, tem-se

$$
\mathcal{E}\{Y(k) \mid \theta(k)=i, \theta(0)=j, Y(0)=M\}>\lambda I .
$$

Os resultados acima podem ser extendidos para o intervalo de tempo $K_{1} \leq k \leq \infty$ em função da suposição de que o filtro é homogêneo no tempo e do fato de $h_{k}, \beta$ e $\lambda$ dados no Teorema 2 serem independentes de $Y(0)$.

Corolário 5.2 (Positividade do processo de erro) Seja $Y(k)$ a matriz de covariância do 
erro de estimação de qualquer filtro linear. Se $(A, B, P)$ é M-alcançável, então existe $\lambda \in \mathbb{R}_{+}$, tal que para toda distribuição inicial $\pi=\pi(0)$,

1. $\mathcal{E}(Y(k+t) \mid \theta(k+t)=i, Y(t)=M)>\lambda I, \forall M \in \mathcal{M}^{n 0}, i \in \mathcal{S}_{\ell}, k \geq K_{1}, t \geq 0$.

2. $\mathcal{E}\{Y(k) \mid \theta(k)=i\}>\lambda I, i \in \mathcal{S}_{\ell}, k \geq K_{1}$.

3. $\mathcal{E}\left\{\sum_{\ell=t}^{t+k-1} Y_{\ell}^{k-1}\left(0,0, \Upsilon_{\ell-1}(D, B)\right) \mid \theta(k)\right\} \geq \lambda I, t \geq 0, k \geq K_{1}$.

Demonstração: Pela homogeneidade no tempo (2.11), podemos escrever,

$$
\begin{aligned}
& \mathcal{E}(Y(k+1) \mid \theta(k+t)=i, \theta(t)=j, Y(t)=M) \\
&=\mathcal{E}(Y(k+1) \mid \theta(k)=i, \theta(0)=j, Y(0)=M), t \geq 0
\end{aligned}
$$

e o Corolário 5.1 implica

$$
\mathcal{E}(Y(k+t) \mid \theta(k+t)=i, \theta(t)=j, Y(t)=M)>\kappa I,
$$

$\log 0$

$$
\mathcal{E}(\mathcal{E}(Y(k+t) \mid \theta(k+t)=i, \theta(t), Y(t)) \mid \theta(k+t)=i, Y(t))>\kappa I,
$$

concluindo a prova do item 1. A segunda afirmação segue de 1 , fazendo $t=0$ e tomando o valor esperado condicionado a $\theta(k)=i$. Quanto a terceira afirmação, temos da definição recursiva em (2.13),

$$
\mathcal{E}\left\{\sum_{\ell=t}^{t+k-1} Y_{\ell}^{k-1}\left(0,0, \Upsilon_{\ell-1}(D, B)\right) \mid \theta(k+t)\right\}=\mathcal{E}\{Y(k+t) \mid \theta(k+t), Y(t)=0\}
$$

tomando $M=0$, a primeira afirmação conclui o resultado.

Observação 3 Note que $Y(t)$ carrega informações sobre a cadeia de Markov e em particular sobre $\pi(t)$. No lado esquerdo de (5.4) queremos mudar o valor de $Y(t)$ sem mudar a distribuição da cadeia de Markov no intervalo $[t, k+t]$, o que explica a introdução do valor esperado condicionado a $\theta(t)=j$. Nas avaliações realizadas nesta etapa, é necessário considerar o valor esperado condicionado à $\theta(t)=j$ e à $\theta(0)=j$.

O próximo resultado segue tomando o valor esperado (não condicionado) na afirmação 1. do Corolário 5.2, 
Corolário 5.3 Seja $Y(k)$ a matriz de covariância do erro de estimação de qualquer filtro linear. Se $(A, B, P)$ é M-alcançável, então existe $\lambda \in \mathbb{R}_{+}$, tal que

$$
\mathcal{E}\{Y(k)\}>\lambda I, k \geq K_{1}
$$

\subsection{M-alcançabilidade e cadeias ergódicas}

A razão pela qual as avaliações para o processo de erro em média dadas no Teorema 2 e Corolário 5.2 estão restritas à $\theta(k)=i \in R_{\pi}$ é dada pela relação com a Proposição 4.1, cujos resultados são válidos para estados de Markov alcançáveis e recorrentes. Os métodos e argumentos de prova desta seção podem ser reproduzidos para estados gerais $\theta(k) \in \mathcal{S}$ sob a condição abaixo.

Suposição 2 Assuma que exista $\beta$ tal que, para todo $k \geq K_{1}$ e $i \in \mathcal{S}$,

$$
\mathcal{E}\left\{X^{k}(B, 0) \mid \theta(k)=i\right\}>\beta I .
$$

Corolário 5.4 Considere a Suposição 2 e a matriz de covariâcia do erro de estimação $Y(k)$ definida em (2.10) para filtros lineares para SLSM. Então, existe $\lambda \in \mathbb{R}_{+}$, tal que, para toda distribuição inicial $\pi=\pi(0)$,

1. $\mathcal{E}\{Y(k) \mid \theta(k)=i\}>\lambda I, i \in \mathcal{S}, k \geq K_{1}$.

2. $\mathcal{E}\left\{\sum_{\ell=t}^{t+k-1} Y_{\ell}^{k-1}\left(0,0, \Upsilon_{\ell-1}(D, B)\right) \mid \theta(k)=i\right\}>\lambda I, t \geq 0, i \in \mathcal{S}, k \geq K_{1}$.

A Suposição 2 pode ser verificada para instantes de tempo finitos calculando as matrizes $S_{i}$ dadas em (4.2) e usando o Lema 4.1. É suficiente que $D_{i}$ seja de posto completo para que a Suposição 2 seja verdadeira, para todo $i \in \mathcal{S}$. Além disso, é trivialmente satisfeita para sistemas alcançáveis com cadeia de Markov ergódicas, neste caso $R_{\pi} \equiv \mathcal{S}$.

\subsubsection{Exemplos ilustrativos}

Abaixo, apresentamos exemplos ilustrando os resultados do Corolário 4.1 e Corolário 5.3. Implementamos o filtro de Kalman em todos os casos, o qual satisfaz as condições de homogeneidade. Obtivemos as estimativas empregando simulação Monte Carlo com 1000 realizações. 
Exemplo 5.1 [M-alcançável] Considere o Exemplo 3.1 com $\Psi=0$ e seja $v=\left[\begin{array}{lll}0 & 0 & 1\end{array}\right]^{\prime}$. A Figura 5.1 ilustra que os valores de $v \mathcal{E}\{X(k)\} v^{\prime}$ e $v \mathcal{E}\{Y(k)\} v^{\prime}$ permanecem positivos com a variação do tempo, exemplificando a suficiência da M-alcançabilidade para positividade em ambos os casos.
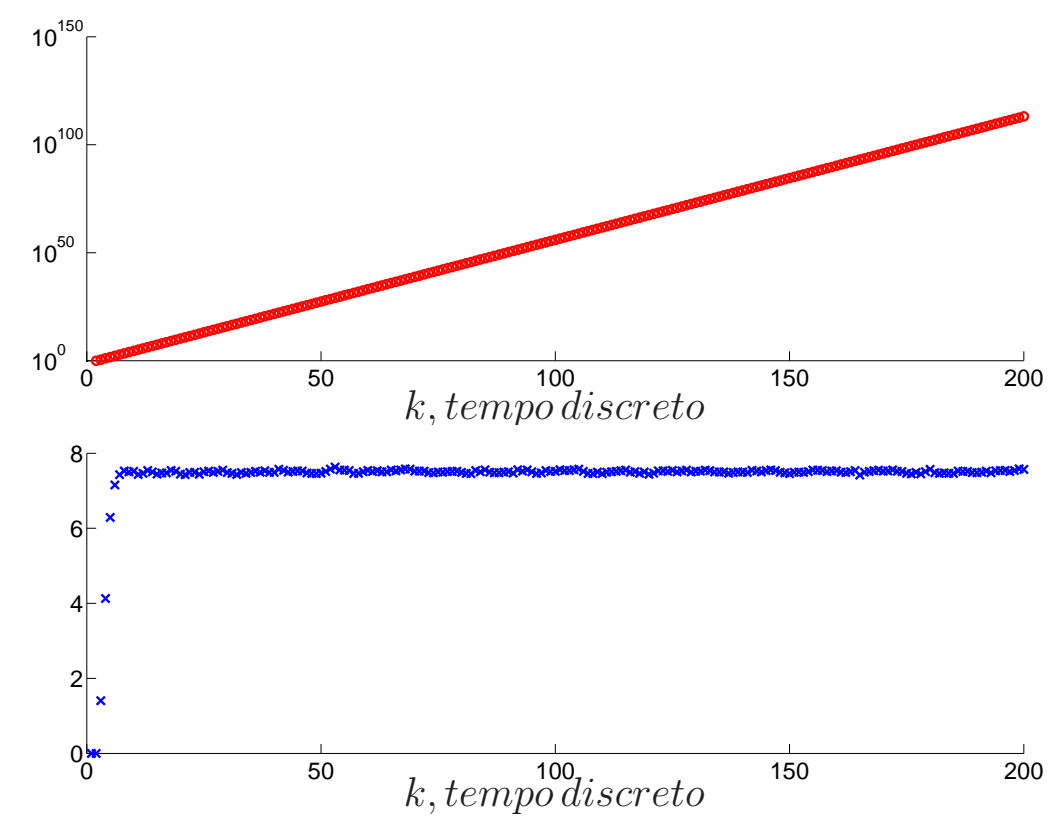

Figura 5.1: Calculo dos valores $v \mathcal{E}\{X(k)\} v^{\prime}$ (denotada com o) e covariância do erro de estimação $v \mathcal{E}\{Y(k)\} v^{\prime}$ (denotada por $\times$ )

Exemplo 5.2 [Não M-alcançável] Seja $v=\left[\begin{array}{lll}0 & 0 & 1\end{array}\right]^{\prime}$. Considere o sistema $\Phi_{M}$ em (2.4) com

$$
\begin{gathered}
A_{1}=\left[\begin{array}{ccc}
0,9 & 0,1 & 0 \\
0 & 1,1 & 0 \\
0 & 0 & 0,9
\end{array}\right], A_{2}=\left[\begin{array}{ccc}
0,9 & 0,1 & 0 \\
0,1 & 0,1 & 0 \\
0 & 0 & 0,8
\end{array}\right], B_{1}=\left[\begin{array}{lll}
0 & 0,1 & 0
\end{array}\right]^{\prime}, B_{2}=0, \\
C_{1}=C_{2}=D_{1}=D_{2}=I, \Psi=\left[\begin{array}{ccc}
0,2 & 0,1 & 0 \\
0,1 & 0 & 0 \\
0 & 0 & 2
\end{array}\right], P=\left[\begin{array}{ll}
0,5 & 0,5 \\
0,5 & 0,5
\end{array}\right] .
\end{gathered}
$$

O teste do posto fornece que $(A, B, P)$ não é M-alcançável. A Figura 5.2 ilustra que os valores de $\mathcal{E}\{X(k)\}$ e $\mathcal{E}\{Y(k)\}$ decrescem com o tempo, exemplificando a necessidade da $M$-alcançabilidade para garantir positividade de $\mathcal{E}\{X(k)\}$, Corolário 4.1. Este resultado e a relação entre $X$ e $Y$ discutida neste capítulo sugerem que M-alcançabilidade é também 
condição necessária para a positividade de $\mathcal{E}\{Y(k)\}$. O exemplo reforça esta linha, observe a figura.
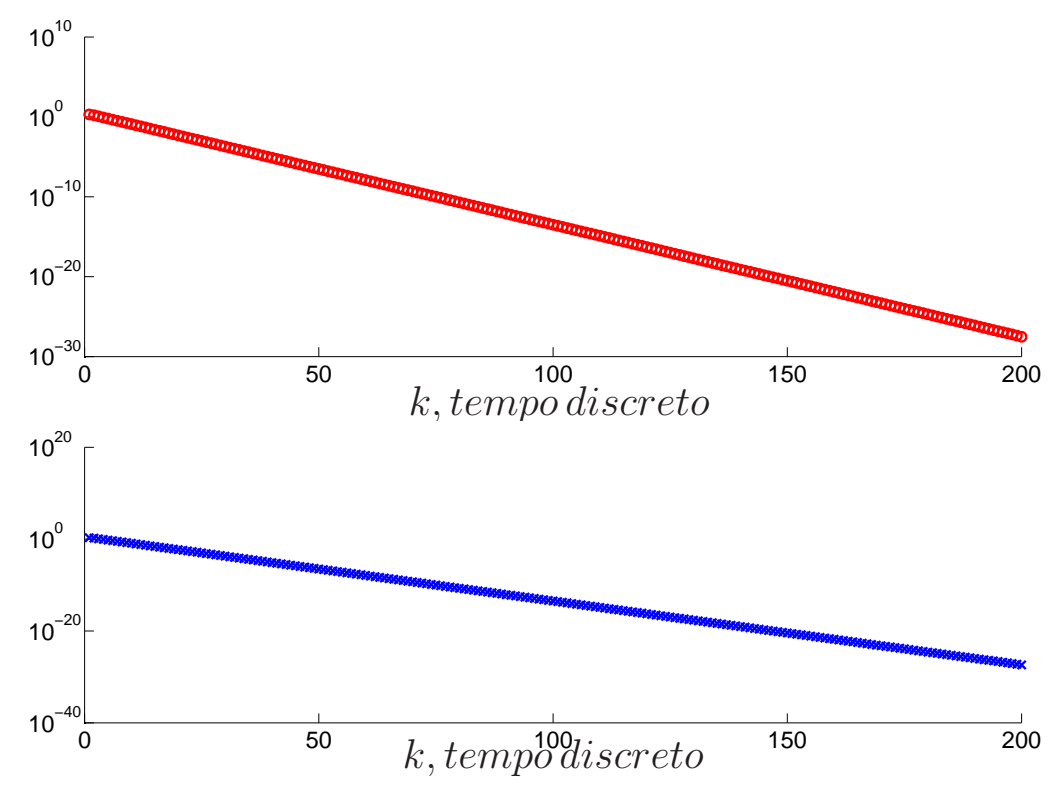

Figura 5.2: Cálculo dos valores de $v \mathcal{E}\{X(k)\} v^{\prime}$ (denotada com o) e covariância do erro de estimação $v \mathcal{E}\{Y(k)\} v^{\prime}$ (denotada por $\times$ ) 


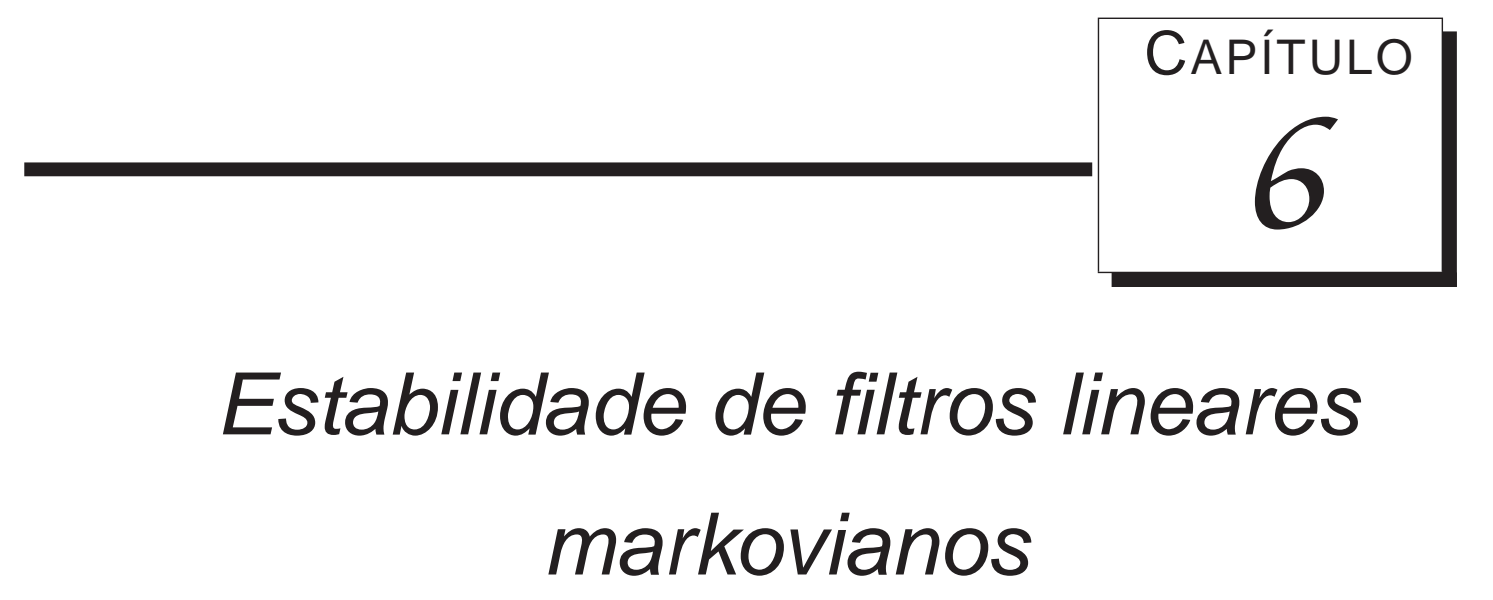

A estabilidade de um filtro é um aspécto relevante para aplicações, uma vez que o modelo da planta sempre apresenta algum nível de imprecisão ou aproximação, afetando o cálculo dos ganhos do filtro. Por exemplo, algumas vezes os valores de $B$ e $\Psi$ não estão disponíveis e o filtro é computado em função de valores nominais em substituição dos valores reais. E em outras aplicações, essas matrizes de parâmetros são manuseados com precisão finita em um computador, ou mudam durante o funcionamento do sistema devido a mudanças ambientais imprevisíveis, falhas, etc. Abrangendo todas estas situações, em nosso estudo o ganho do filtro é computado baseando-se nos parâmetros $\Sigma$ e $E$ (em substituição de $\Psi$ e $B$, respectivamente) e aplicado no sistema atual cujos parâmetros $\Psi$ e $B$ estão sujeitos a mudanças de qualquer magnitude. Nos referimos a $\Sigma, E$ e grandezas relacionadas a estes como teóricas, em oposição à grandezas reais que estão relacionadas com os valores $\Psi$ e $B$.

Neste capítulo mostramos que as Condições 1 e 2 garantem a estabilidade de estimadores lineares markovianos. O conceito de estabilidade tratada aqui é definida como a invariância (aos parâmetros das matrizes de ruído aditivo) da existência de limitantes superiores de $Y$. Esta noção de estabilidade está em perfeita analogia com a estabilidade de um sistema na forma padrão $x(k+1)=A x(k)+w(k)$ onde $w(k)$ é um processo de ruído i.i.d. e os autovalores de $A$ pertencem à um disco unitário se, e somente se, para cada matriz de covariância 
de $w$, existe um limitante superior para $\mathcal{E}\left\{\|x(k)\|^{2}\right\}$.

Resultados preliminares são apresentatos em [36] e [39], os quais abordam o filtro de Kalman para SLSM para cadeias ergódicas e perturbações somente na covariância da condição inicial. Adicionalmente a referência emprega uma conjectura provada aqui, Lema 6.1.

Assuma que os valores de $\Psi$ e de $B$ não estejam disponíveis para a implementação do filtro, logo empregam-se valores nominais $\Sigma$ e $E$ em substituição dos verdadeiros. Assim, adotamos dois modelos para análise. O primeiro sendo o modelo verdadeiro e também conhecido como modelo real, o qual é caracterizado pelas matrizes de ruído aditivo de estado e covariância inicial denotadas, respectivamente, por $B$ e $\Psi$. O segundo sendo o modelo nominal, no qual consideramos as informações disponíveis e através do qual realizamos o cálculo do ganho do filtro. Denotamos as matrizes de ruído e covariância inicial respectivamente por $E$ e $\Sigma$. Por consequência assumiremos as Condições 1 e 2 nas variáveis $E$ e $\Sigma$, por exemplo, consideramos que $(A, E, \Sigma)$ seja M-alcançável.

No sentido discutido acima, consideramos a seguinte definição de estabilidade do filtro.

Definição 6.1 (Estabilidade) Dizemos que um filtro linear markoviano é estável se, para cada $B \in \mathcal{N}^{N, n, p}$ e $\Psi \in \mathcal{M}^{n 0}$, existir $\bar{Y} \in \mathcal{M}^{n+}$ tal que $\mathcal{E}\left\{Y^{k}(D, B, \Psi)\right\}<\bar{Y}, k \geq 0$.

Observe que não é possível comparar (admitir algum tipo de ordenação) de forma direta os valores de $\mathcal{E}\left\{Y_{t}^{k}(D, E, \Sigma)\right\}$ (limitado por hipótese, Suposição 1) e $\mathcal{E}\left\{Y_{t}^{k}(D, B, \Psi)\right\}$, ou seja, uma grande dificuldade é encontrar um escalar positivo $\vartheta$ que satisfaça

$$
\vartheta \mathcal{E}\left\{Y_{t}^{k}(D, B, \Psi) \leq \mathcal{E}\left\{Y_{t}^{k}(D, E, \Sigma)\right\} \leq \bar{P}, k \geq 0\right.
$$

O método para tratar este ponto é direto. Criamos uma trajetória auxiliar $\mathcal{E}\left\{Y_{t}^{k}(D, I, \Sigma) \mid \theta(k)=i\right\}$, que possa ser comparada com ambas as covariâncias de erro $\mathcal{E}\left\{Y_{t}^{k}(D, B, \Psi) \mid \theta(k)=i\right\}$ e $\mathcal{E}\left\{Y_{t}^{k}(D, E, \Sigma) \mid \theta(k)=i\right\}$, cuja limitação é proveniente da Suposição 1. Para tanto, mostramos a existência de uma recursão que satisfaça (6.1), que limita inferiormente o processo de erro nominal $\mathcal{E}\left\{Y_{t}^{k}(D, E, \Sigma)\right\}$ no intervalo $[t, k]$, Corolário 6.1. E através de um resultado auxiliar apresentado no Lema 6.1, extendemos este resultado para limitação inferior uniforme de $\mathcal{E}\left\{Y_{t}^{k}(D, E, \Sigma) \mid \theta(k)=i\right\}$.

O próximo Corolário caracteriza o discutido acima para o intervalo de tempo $[t, k]$. 
Corolário 6.1 Considere a Suposição 2 e o processo de erro $\{Y(k)\}$. Para cada $t \geq 0, k \geq$ $K_{1}+t$, existe $\xi>0$ tal que

$$
\xi^{2} \mathcal{E}\left\{Y_{t}^{k}(D, I, \Psi) \mid \theta(k)=i\right\} \leq \mathcal{E}\left\{Y_{t}^{k}(D, B, \Psi) \mid \theta(k)=i\right\}
$$

Demonstração: Segue de (2.13) e do Corolário 5.2 (iii) que

$$
\mathcal{E}\left\{\sum_{\ell=t}^{k-1} Y_{\ell}^{k-1}\left(0,0, \Upsilon_{\ell-1}(D, E)\right) \mid \theta(k)\right\} \geq \lambda I, t \geq 0, k \geq K_{1}+t
$$

Por outro lado, para cada $k \geq K_{1}+t$ sempre existe um escalar suficientemente pequeno $\xi>0$, satisfazendo

$$
\xi^{2} \mathcal{E}\left\{\sum_{\ell=t}^{k-1} Y_{\ell}^{k-1}\left(0,0, \Upsilon_{\ell-1}(D, I)\right) \mid \theta(k)=i\right\} \leq \lambda I
$$

então tomando $\xi<1$ e empregando (2.13), (2.15), (6.2), (6.3), obtemos

$$
\begin{aligned}
& \xi^{2} \mathcal{E}\left\{Y_{t}^{k}(D, I, \Sigma) \mid \theta(k)=i\right\}= \\
&=\xi^{2} \mathcal{E}\left\{Y_{t}^{k-1}(0,0, \Sigma) \mid \theta(k)=i\right\}+\xi^{2} \mathcal{E}\left\{\sum_{\ell=t+1}^{k-1} Y_{\ell}^{k-1}\left(0,0, \Upsilon_{\ell-1}(D, I)\right) \mid \theta(k)=i\right\} \\
& \quad \leq \mathcal{E}\left\{Y_{t}^{k-1}(0,0, \Sigma) \mid \theta(k)=i\right\}+\lambda I \\
& \quad \leq \mathcal{E}\left\{Y_{t}^{k-1}(0,0, \Sigma) \mid \theta(k)=i\right\}+\mathcal{E}\left\{\sum_{\ell=t+1}^{k-1} Y_{\ell}^{k-1}\left(0,0, \Upsilon_{\ell-1}(D, E)\right) \mid \theta(k)=i\right\} \\
&= \mathcal{E}\left\{Y_{t}^{k}(D, E, \Sigma) \mid \theta(k)=i\right\},
\end{aligned}
$$

A seguir escrevemos $\mathcal{E}\left\{Y_{0}^{k}\right\}=\mathcal{E}\left\{Y_{s}^{k}(0,0, \eta)\right\}$, onde $\eta=\mathcal{E}\left\{Y_{0}^{k}\right\}$, nos permitindo manipular o valor esperado de $Y$ recursivamente nos passos seguintes. O resultado apresentado no Lema 6.1 passou por um processo de adequação desde o início do estudo. Inicialmente o apresentamos como uma conjectura, [39], em seguida obtivemos uma prova deste resultado no estudo da estabilidade do filtro de Kalman para SLSM no caso em que a distribuição da cadeia é positiva, veja [40]. Neste trabalho, ajustamos o resultado com o acréscimo do valor esperado condicionado, o qual possibilita a extensão do Corolário 6.1 para limitação uniforme e qualquer $k \geq K_{1}$. A prova é dada pelo desenvolvimento do valor esperado, pelo 
Teorema da probabilidade total e pela propriedade de observação do estado disreto $\theta(k)$. O resultado fornece uma relação entre a recursão em função do valor esperado discutida acima e o processo de erro geral.

Lema 6.1 Considere Y como definido em (2.10) $e$

$$
\eta=\mathcal{E}\left\{Y^{s}(D, E, \Sigma) \mid \theta(s)\right\}, s \geq 0
$$

então vale o seguinte para $k \geq s$,

$$
\mathcal{E}\left\{Y_{s}^{k}\left(0,0, Y^{s}(D, E, \Sigma)\right) \mid \theta(k)\right\}=\mathcal{E}\left\{Y_{s}^{k}(0,0, \eta) \mid \theta(k)\right\}
$$

Demonstração: Tomando $\Gamma_{i}=\left(A_{i}+H_{i}(k) C_{i}\right)$ e usando a lei da probabilidade total, escrevemos

$$
\begin{gathered}
\mathcal{E}\left\{Y_{s}^{k}\left(0,0, Y^{s}(D, E, \Sigma)\right) \mid \theta(k)\right\}=\sum_{i_{(k-1)}=1}^{N} p_{i_{(k-1)} \theta(k)} \Gamma_{i_{(k-1)}}\left(\sum_{i_{(k-2)}=1}^{N} p_{i_{(k-2)} i_{(k-1)}} \Gamma_{i_{(k-2)}} \ldots \times\right. \\
\left(\sum_{i_{(s+1)}=1}^{N} p_{i_{(s+1)} i_{(s+2)}} \Gamma_{i_{(s+1)}} \sum_{i_{s}=1}^{N} \mathcal{E}\left\{Y^{s}(D, E, \Sigma) 1_{\left\{\theta(s)=i_{s}\right\}} \mid \theta(k)\right\} \Gamma_{i_{(s+1)}}^{\prime}\right) \ldots \Gamma_{1_{(k-1)}}^{\prime} .
\end{gathered}
$$

Similarmente, expandindo $\mathcal{E}\left\{Y_{s}^{k}(0,0, \eta) \mid \theta(k)\right\}$ obtém-se

$$
\begin{aligned}
& \mathcal{E}\left\{Y_{s}^{k}(0,0, \eta) \mid \theta(k)\right\}=\sum_{i_{(k-1)}=1}^{N} p_{i_{(k-1)}} \theta(k) \Gamma_{i_{(k-1)}}\left(\sum_{i_{(k-2)}=1}^{N} p_{i_{(k-2)} i_{(k-1)}} \Gamma_{i_{(k-2)}} \ldots \times\right. \\
& \left(\sum_{i_{(s+1)}=1}^{N} p_{i_{(s+1)} i_{(s+2)}} \Gamma_{i_{(s+1)}} \sum_{i_{s}=1}^{N} \mathcal{E}\left\{\mathcal{E}\left\{Y^{s}(D, E, \Sigma) \mid \theta(s)\right\} 1_{\left\{\theta(s)=i_{s}\right\}} \mid \theta(k)\right\} \Gamma_{i_{(s+1)}}^{\prime}\right) \ldots \Gamma_{1_{(k-1)}}^{\prime}
\end{aligned}
$$

Basta mostrar que as expressões desenvolvidas em (6.5) e (6.6) são equivalentes. Usando propriedades do valor esperado, obtemos

$$
\begin{aligned}
\mathcal{E}\left\{\mathcal{E}\left\{Y^{s}(D, E, \Sigma) \mid \theta(s)\right\}\right. & \left.1_{\{\theta(s)=i\}} \mid \theta(k)\right\} \\
& =\mathcal{E}\left\{\mathcal{E}\left\{Y^{s}(D, E, \Sigma) \mid \theta(s)\right\} \mid \theta(s)=i, \theta(k)\right\} P(\theta(s)=i \mid \theta(k)) \\
& =\mathcal{E}\left\{Y^{s}(D, E, \Sigma) \mid \theta(s)=i\right\} P(\theta(s)=i \mid \theta(k))
\end{aligned}
$$


e da conhecida propriedade da Cadeia de Markov, a qual afirma que os estados passados e futuros são independentes quando é dado o estado presente (lembrando que $Y^{s}(D, E, \Sigma)$ é uma função somente de estados passados $\theta(0), \ldots, \theta(s)$ ), temos que a igualdade acima é equivalente a

$$
\mathcal{E}\left\{Y^{s}(D, E, \Sigma) \mid \theta(s)=i, \theta(k)\right\} P(\theta(s)=i \mid \theta(k))
$$

que é igual a $\mathcal{E}\left\{Y^{s}(D, E, \Sigma) 1_{\{\theta(s)=i\}} \mid \theta(k)\right\}$, completando a prova.

Observação 4 A hipótese markoviana acima é indispensável para o resultado, a qual garante que $\theta(k)$ é a única variável aleatória envolvida em $\Gamma_{i}=\left(A_{i}+H_{i}(k) C_{i}\right)$. Em cenários mais gerais $H(k)$ pode depender de $\theta(0), \ldots, \theta(k)$, e não podemos manipular o valor esperado como em (6.5) (poderia permanecer fora dos somatórios). Além disso, note que o condicional em $\theta(s)$ é também essencial para que a equivalência entre (6.7) e (6.8) seja válida.

A principal ideia desta etapa é obter uma grandeza auxiliar limitada $\xi^{2} \mathcal{E}\left\{Y^{k}(D, I, \Sigma) \mid \theta(k)=i\right\}$ (de uma forma uniforme), que seja um limitante superior para uma variação do processo de erro $\beta^{2} \mathcal{E}\left\{Y^{k}(D, B, \Psi) \mid \theta(k)=i\right\}, \beta>0, k \geq K_{1}$. A questão que surge é como assegurar que a desigualdade

$$
\beta^{2} \mathcal{E}\left\{Y^{k}(D, B, \Psi) \mid \theta(k)=i\right\} \leq \xi^{2} \mathcal{E}\left\{Y^{k}(D, I, \Sigma) \mid \theta(k)=i\right\} \leq \mathcal{E}\left\{Y^{k}(D, E, \Sigma) \mid \theta(k)=i\right\}
$$

permaneça ao longo do tempo para algum $\beta, \xi>0$ e $k \geq K_{1}$. O Lema 6.2 mostra que existe um escalar $\xi \in \mathbb{R}_{+}$(independente de $k$ ) satisfazendo a segunda desigualdade acima, ou seja, satisfazendo o Corolário 6.1 para $k \geq K_{1}$.

Lema 6.2 Considere o processo de erro $Y(k)$ dado em (2.10), então existe $\zeta \in \mathbb{R}_{+}$tal que para todo $k \geq K_{1}$,

$$
\zeta^{2} \mathcal{E}\left\{Y^{k}(D, I, \Sigma) \mid \theta(k)=i\right\} \leq \mathcal{E}\left\{Y^{k}(D, E, \Sigma) \mid \theta(k)=i\right\}
$$


Demonstração: O resultado segue por indução na variável $k$, iniciando com $k=K_{1}$. Inicialmente, definimos $v>0$ (usualmente um escalar pequeno) tal que

$$
v^{2} \mathcal{E}\left\{\sum_{\ell=t}^{t+K_{1}} Y_{\ell}^{k}\left(0,0, \Upsilon_{\ell-1}(D, I)\right) \mid \theta(k)=i\right\} \leq \lambda I, \quad t \geq 0
$$

onde $v$ é independente de $t$ devido à homogeneidade no tempo do processo $Y$ (claro que, $v$ depende do tamanho do intervalo de tempo, $K_{1}$ ) e $\lambda$ é dado como no Corolário 5.2. Considere $\zeta=\sqrt{\min \left(\xi^{2}, v^{2}\right)} \operatorname{com} \xi>0$ como no Corolário 6.1. Voltando à indução, para $k=K_{1} \mathrm{o}$ resultado segue diretamente do Corolário 6.1,

$\zeta^{2} \mathcal{E}\left\{Y^{k}(D, I, \Sigma) \mid \theta(k)=i\right\} \leq \xi^{2} \mathcal{E}\left\{Y^{k}(D, I, \Sigma) \mid \theta(k)=i\right\} \leq \mathcal{E}\left\{Y^{k}(D, E, \Sigma) \mid \theta(k)=i\right\}$

Por hipótese de indução assumimos que, para algum $k=\bar{k}>K_{1}$ fixo,

$$
\left.\zeta^{2} \mathcal{E}\left\{Y^{s}(D, I, \Sigma) \mid \theta(s)=i\right\} \leq \mathcal{E}\left\{Y^{s}(D, E, \Sigma) \mid \theta(s)=i\right\}, \quad \forall K_{1} \leq s \leq \bar{k}\right]
$$

Com o objetivo de completar a indução, assumimos que (6.10) seja válido para $k=\bar{k}+1$. Por facilidade de notação, no restante desta prova, denotaremos $k_{0}=\bar{k}+1-K_{1}$,

$$
\mu=\zeta^{2} \mathcal{E}\left\{Y^{k_{0}}(D, I, \Sigma) \mid \theta\left(k_{0}\right)\right\}
$$

$\mathrm{e}$

$$
\eta=\mathcal{E}\left\{Y^{k_{0}}(D, E, \Sigma) \mid \theta\left(k_{0}\right)\right\}
$$

Como $k_{0} \in\left[K_{1}, \bar{k}\right]$, a desigualdade 6.10 implica $\mu \leq \eta$. Considerando a definição recursiva de $Y^{k}(D, E, \Sigma)$ como em (2.13) - (2.15) escrevemos

$$
\begin{aligned}
& \zeta^{2} \mathcal{E}\left\{Y^{k}(D, I, \Sigma) \mid \theta(k)=i\right\}=\zeta^{2} \mathcal{E}\left\{Y_{k_{0}}^{k}\left(D, I, Y^{k_{0}}(D, I, \Sigma)\right) \mid \theta(k)=i\right\} \\
& =\mathcal{E}\left\{Y_{k_{0}}^{k}\left(0,0, \zeta^{2} Y^{k_{0}}(D, I, \Sigma)\right) \mid \theta(k)=i\right\}+\zeta^{2} \mathcal{E}\left\{\sum_{\ell=k_{0}+1}^{k} Y_{\ell}^{k}\left(0,0, \Upsilon_{\ell-1}(D, I)\right) \mid \theta(k)=i\right\}
\end{aligned}
$$


e aplicando o Lema 6.1,

$$
\begin{aligned}
\zeta^{2} \mathcal{E} & \left\{Y^{k}(D, I, \Sigma) \mid \theta(k)=i\right\}= \\
& =\mathcal{E}\left\{Y_{k_{0}}^{k}(0,0, \mu) \mid \theta(k)=i\right\}+\zeta^{2} \mathcal{E}\left\{\sum_{\ell=k_{0}+1}^{k} Y_{\ell}^{k}\left(0,0, \Upsilon_{\ell-1}(D, I)\right) \mid \theta(k)=i\right\} .
\end{aligned}
$$

Como o intervalo de tempo da soma acima é $K_{1}$, podemos aplicar a equação (6.9); Isto em adição com $\mu \leq \eta$ leva a

$$
\zeta^{2} \mathcal{E}\left\{Y^{k}(D, I, \Sigma) \mid \theta(k)=i\right\} \leq \mathcal{E}\left\{Y_{k_{0}}^{k}(0,0, \eta) \mid \theta(k)=i\right\}+\lambda I
$$

Do Corolário 5.2 (iii) obtém-se

$$
\begin{aligned}
\zeta^{2} \mathcal{E} & \left\{Y^{k}(D, I, \Sigma) \mid \theta(k)=i\right\} \\
& \leq \mathcal{E}\left\{Y_{k_{0}}^{k}(0,0, \eta) \mid \theta(k)=i\right\}+\mathcal{E}\left\{\sum_{\ell=k_{0}+1}^{k} Y_{\ell}^{k}\left(0,0, \Upsilon_{\ell-1}(D, E)\right) \mid \theta(k)=i\right\}
\end{aligned}
$$

empregando novamente (2.13), (2.15) e o Lema 6.1 (na ordem inversa ao usado acima),

$$
\begin{aligned}
& \zeta^{2} \mathcal{E}\left\{Y^{k}(D, I, \Sigma) \mid \theta(k)=i\right\} \\
& \leq \mathcal{E}\left\{Y_{k_{0}}^{k}\left(0,0, Y^{k_{0}}(D, E, \Sigma)\right) \mid \theta(k)=i\right\}+\mathcal{E}\left\{\sum_{\ell=k_{0}+1}^{k} Y_{\ell}^{k}\left(0,0, \Upsilon_{\ell-1}(D, E)\right) \mid \theta(k)=i\right\} \\
& =\mathcal{E}\left\{Y_{k_{0}}^{k}\left(D, E, Y^{k_{0}}(D, E, \Sigma)\right) \mid \theta(k)=i\right\}=\mathcal{E}\left\{Y^{k}(D, E, \Sigma) \mid \theta(k)=i\right\}
\end{aligned}
$$

e a prova está completa.

Os resultados apresentados acima dão base para a prova do principal resultado do capítulo, a Suposição 2 é suficientes para a estabilidade de filtros markovianos. A estratégia de prova segue a mesma linha do Lema 6.2, mas neste caso, usamos uma variação do processo de erro $\mathcal{E}\left\{Y^{k}(D, B, \Psi) \mid \theta(k)=i\right\}$ como limitante superior do processo $\mathcal{E}\left\{Y^{k}(D, I, \Sigma) \mid \theta(k)=i\right\}$, que por sua vez é limitada superiormente.

Teorema 3 (Estabilidade) Considere $Y$ como definido em (2.10), e suponha que as Condições 1 e 2 sejam válidas. Então existe $\bar{Y} \in \mathcal{M}^{n+}$ tal que

$$
\mathcal{E}\left\{Y^{k}(D, E, \Sigma) \mid \theta(k)=i\right\}<\bar{Y}, \forall k \geq K_{1} .
$$


Demonstração: Seja $\zeta>0$ como no Lema 6.2. Podemos escolher um escalar positvo $\beta<\zeta$ tal que para todo $k \geq 0, i \in \mathcal{S}$,

$$
\beta B_{i}(k) B_{i}(k)^{\prime} \leq \zeta I
$$

e

$$
\beta \Psi \leq \zeta \Sigma,
$$

onde $\zeta$ é como no Lema 6.2. Então empregando (2.13) e (6.11) - (6.12), podemos escrever

$$
\begin{aligned}
& \beta^{2} \mathcal{E}\left\{Y^{k}(D, B, \Psi) \mid \theta(k)=i\right\} \\
& =\beta^{2} \mathcal{E}\left\{Y^{k}(0,0, \Psi) \mid \theta(k)=i\right\}+\beta^{2} \mathcal{E}\left\{\sum_{\ell=1}^{k} Y_{\ell}^{k}\left(0,0, \Upsilon_{\ell-1}(D, B)\right) \mid \theta(k)=i\right\} \\
& \leq \zeta^{2} \mathcal{E}\left\{Y^{k-1}(0,0, \Sigma) \mid \theta(k)=i\right\}+\zeta^{2} \mathcal{E}\left\{\sum_{\ell=1}^{k} Y_{\ell}^{k}\left(0,0, \Upsilon_{\ell-1}(D, I)\right) \mid \theta(k)=i\right\} \\
& =\zeta^{2} \mathcal{E}\left\{Y^{k}(D, I, \Sigma) \mid \theta(k)=i\right\} .
\end{aligned}
$$

O Lema 6.2 e a Suposição 1 implicam

$$
\begin{aligned}
\beta^{2} \mathcal{E}\left\{Y^{k}(D, B, \Psi) \mid \theta(k)=i\right\} & \leq \zeta^{2} \mathcal{E}\left\{Y^{k}(D, I, \Sigma) \mid \theta(k)=i\right\} \\
& \leq \mathcal{E}\left\{Y^{k}(D, E, \Sigma) \mid \theta(k)=i\right\} \leq \bar{P}, k \geq K_{1}
\end{aligned}
$$

portanto $\mathcal{E}\left\{Y^{k}(D, B, \Psi) \mid \theta(k)=i\right\} \leq \beta^{-2} \bar{P}=\delta \bar{P}=\bar{Y}$, completando a prova.

O próximo corolário segue diretamente de propriedades do valor esperado

$$
\mathcal{E}\left\{\mathcal{E}\left\{Y^{k}(D, B, \Psi) \mid \theta(k)=i\right\}\right\} \leq \mathcal{E}\{\bar{Y}\}
$$

Corolário 6.2 Considere $Y$ como definido em (2.10), e suponha que as Condições 1 e 2 sejam válidas. Se $(A, E, P)$ é M-alcançável então existe $\bar{Y} \in \mathcal{M}^{n+}$ tal que $\mathcal{E}\left\{Y^{k}(D, B, \Psi)\right\}<\bar{Y}, \forall k \geq K_{1}$. 


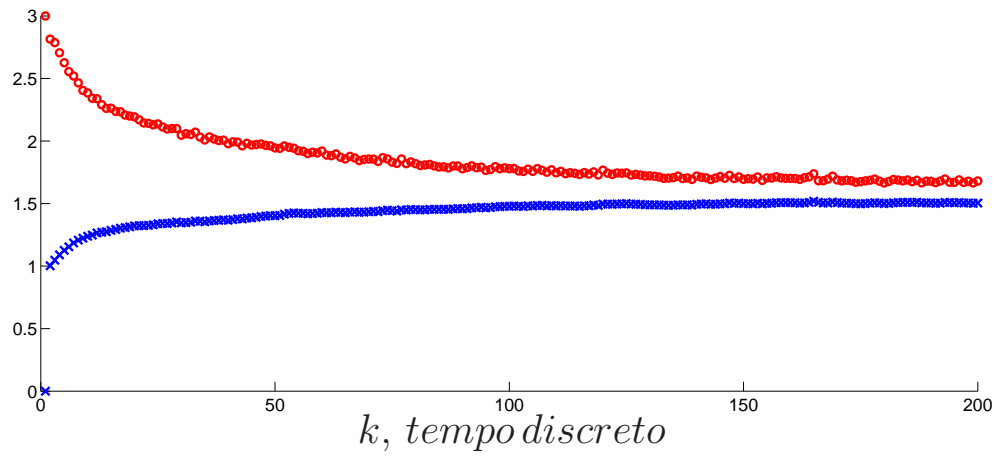

Figura 6.1: Estimação da Covariância de erro de Exemplo 6.1. $\mathcal{E}\left\{Y^{k}(D, E, \Sigma)\right\}$ (denotada com o) e $\mathcal{E}\left\{Y^{k}(D, B, \Psi)\right\}$ (detotada com $\times$ ).

\subsection{Exemplos ilustrativos}

Nesta seção apresentamos exemplos ilustrativos do resultado de estabilidade. Os casos apresentados são bastante simples, de forma que buscamos ilustrar de forma bastante direta que os resultados apresentos são válidos. Variamos as Condições 1 e 2 em cada um dos exemplos, de forma que quando uma das condições é falha, a estabilidade não ocorre. Ilustramos também que a Suposição 2 não é necessária para estabilidade. Implementamos o ELMQ como apresentado em [18], [27], [30] e [23].

Exemplo 6.1 (Suposição 1, Suposição 2) Considere o sistema $\Phi_{M}$ com

$$
\begin{gathered}
A_{1}=\left[\begin{array}{ccc}
0 & 0 & 0 \\
0 & 0,1 & 1 \\
0 & 0 & 0,9
\end{array}\right], A_{2}=\left[\begin{array}{ccc}
0,9 & 0 & 0 \\
0,1 & 0,9 & 0 \\
0 & 0 & 0,9
\end{array}\right], E_{1}=\left[\begin{array}{lll}
1 & 0 & 0
\end{array}\right]^{\prime}, \\
E_{2}=\left[\begin{array}{lll}
1 & 0 & 0
\end{array}\right]^{\prime}, B_{1}=\left[\begin{array}{ccc}
1 & 0 & 0
\end{array}\right]^{\prime}, B_{2}=\left[\begin{array}{lll}
1 & 0 & 1
\end{array}\right]^{\prime}, \\
C_{1}=C_{2}=D_{1}=D_{2}=I, \Sigma=0, \Psi=\left[\begin{array}{lll}
1 & 0 & 0 \\
0 & 1 & 0 \\
0 & 0 & 1
\end{array}\right], P=\left[\begin{array}{ll}
0,9 & 0,1 \\
0,9 & 0,1
\end{array}\right] .
\end{gathered}
$$

Comcluímos através do Teorema do posto completo que $(A, B, P)$ é M-alcançável, e nas condições do sistema, a Suposição 2 é satisfeita. Computamos os ganhos do ELMQ em função dos parâmetros nominais $E, \Sigma$. Os resultados indicam que $\mathcal{E}\left\{Y^{k}(D, E, \Sigma)\right\} e$ $\mathcal{E}\left\{Y^{k}(D, B, \Psi)\right\}$ são limitados, (veja a Figura 6.1), sugerindo que o filtro é estável. 


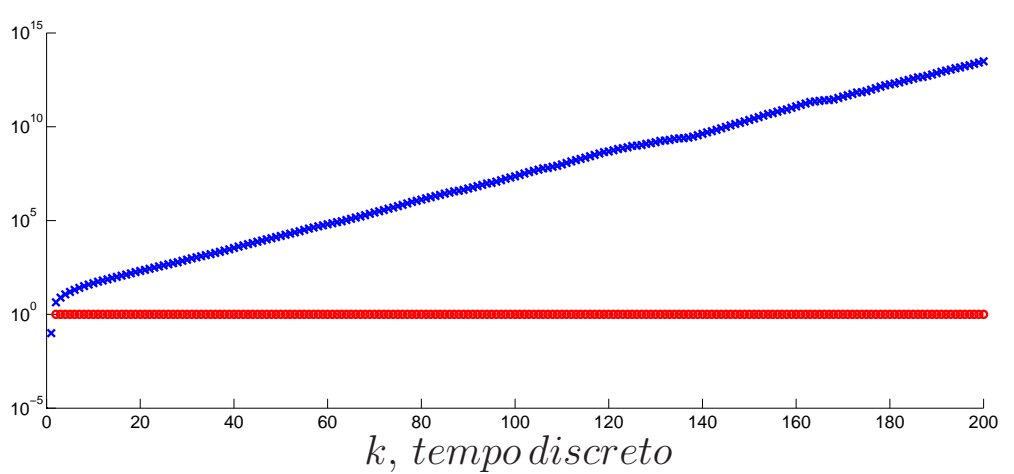

Figura 6.2: Estimação da Covariância de erro de Exemplo 6.2. $\mathcal{E}\left\{Y^{k}(D, E, \Sigma)\right\}$ (denotada com $\circ)$ e $\mathcal{E}\left\{Y^{k}(D, B, \Psi)\right\}$ (detotada com $\left.\times\right)$.

Exemplo 6.2 (Suposição 1 falha, Suposição 2) Considere o sistema $\Phi_{M}$ do Exemplo 6.1 com

$$
\begin{gathered}
A_{1}=\left[\begin{array}{ccc}
0 & 0 & 0 \\
0 & 1,1 & 0 \\
0 & 0 & 1
\end{array}\right], A_{2}=\left[\begin{array}{ccc}
0 & 0,1 & 0 \\
0 & 0,9 & 0 \\
0 & 0 & 1
\end{array}\right], E_{1}=\left[\begin{array}{ccc}
1 & 1,5 & 1
\end{array}\right]^{\prime}, \\
E_{2}=\left[\begin{array}{lll}
1 & 1 & 1
\end{array}\right]^{\prime}, B_{1}=B_{2}=\left[\begin{array}{lll}
1 & 0 & 0
\end{array}\right]^{\prime}, C_{1}=C_{2}=D_{1}=D_{2}=I, \\
\Sigma=\left[\begin{array}{ccc}
0 & 0,1 & 0 \\
0 & 0 & 0 \\
0 & 0 & 0,1
\end{array}\right], \Psi=0, P=\left[\begin{array}{cc}
0,9 & 0,1 \\
0,5 & 0,5
\end{array}\right] .
\end{gathered}
$$

Temos que $(A, E, P)$ é M-alcançável. Os cálculos do ganho do ELMQ foram realizados em função dos valores E, $\Sigma$. O exemplo ilustra que o erro de estimação cresce exponencialmente sob perturbações no modelo de ruído, veja a Figura 6.2.

Exemplo 6.3 (Suposição 1, Suposição 2 falha) Considere o sistema $\Phi_{M}$ do Exemplo 6.1 com

$$
\begin{gathered}
A_{1}=\left[\begin{array}{ccc}
0 & 0,1 & 0 \\
0 & 1,1 & 0 \\
0 & 0 & 0
\end{array}\right], A_{2}=\left[\begin{array}{ccc}
0,9 & 0,1 & 0 \\
0,1 & 0 & 0 \\
0 & 0 & 0
\end{array}\right], E_{1}=E_{2}=0, \\
B_{1}=\left[\begin{array}{lll}
0,5 & 0,1 & 0
\end{array}\right]^{\prime}, B_{2}=\left[\begin{array}{lll}
0,5 & 0 & 1,5
\end{array}\right]^{\prime}, P=\left[\begin{array}{ll}
0,1 & 0,9 \\
0,1 & 0,9
\end{array}\right],
\end{gathered}
$$




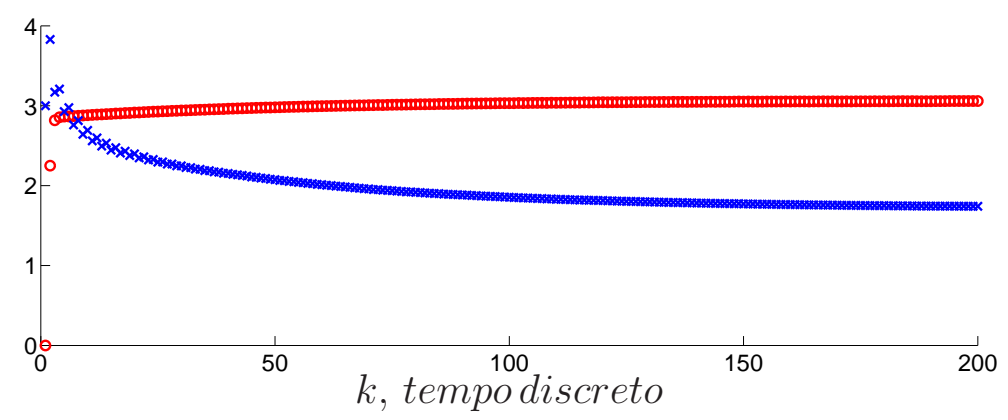

Figura 6.3: Estimação da Covariância de erro de Exemplo 6.2. $\mathcal{E}\left\{Y^{k}(D, E, \Sigma)\right\}$ (denotada com $\circ$ ) $\operatorname{e}\left\{Y^{k}(D, B, \Psi)\right\}$ (detotada com $\times$ ).

$$
\Psi=\left[\begin{array}{ccc}
0,2 & 0,1 & 0 \\
0,1 & 0 & 0 \\
0 & 0 & 1
\end{array}\right], \Sigma=\left[\begin{array}{ccc}
0,5 & 0 & 0 \\
0 & 0,2 & 0 \\
0 & 0 & 0
\end{array}\right]
$$

Obtemos através do teste do posto que $(A, E, P)$ não é M-alcançável. A Figura 6.3 sugere que as grandezas $\mathcal{E}\left\{Y^{k}(D, E, \Sigma)\right\}$ e $\mathcal{E}\left\{Y^{k}(D, B, \Psi)\right\}$ são limitados e portanto o filtro é estável. Este exemplo mostra que a Suposição 2 não é necessária. 


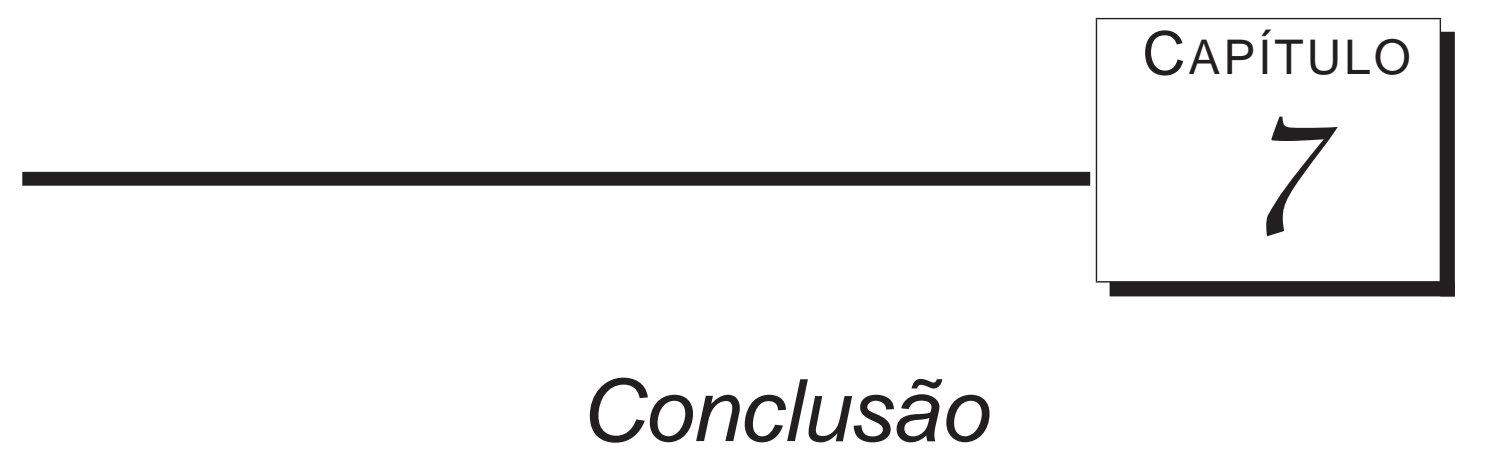

Este trabalho considerou o estudo da alcançabilidade, da positividade uniforme da covariância do processo do erro de estimação e da estabilidade de estimadores para sistemas lineares sujeitos a saltos markovianos.

Inicialmente, introduzimos o conceito de M-alcançabilidade e o conjunto de matrizes $\mathcal{R}$ para SLSM. Obtemos um teste para M-alcançabilidade, Teorema 1, estabelecendo um paralelo com o teste usual de alcançabilidade para sistemas lineares invariantes no tempo.

A noção de alcançabilidade introduzida possibilitou a obtenção de condições de positividade. De fato, mostramos que M-alcançabilidade é uma condição necessária e suficiente para a positividade do segundo momento do estado em média, isto é, $\mathcal{E}\left\{X^{k}(E, \Sigma)\right\}>0$, veja o Lema 4.1, deixando claro o papel da M-alcançabilidade na estrutura de SLSM. Em seguida, mostramos que M-alcançabilidade é condição suficiente para a positividade uniforme do valor esperado do processo de erro, ou seja, existe um escalar positivo $\beta$ tal que $\mathcal{E}\{Y(k)\} \geq \beta I$, Teorema 2 e Corolário 5.2.

A positividade da covariância é a principal ferramenta para o estudo da estabilidade, nos permitindo mostrar que a covariância é limitada em média, mesmo sob perturbações nos parâmetros da planta, como apresentado no Teorema 3. É um pouco paradoxal que seja preciso ruído completo na planta para que se garanta estabilidade do filtro, no entanto é este ruído que evidencia possíveis dinâmicas instáveis durante o cálculo do ganho - sem ruído completo, estas dinâmicas podem ficar desapercebidas. Em aplicações de controle e 
filtragem é preciso estar atento a estes aspectos, pois mesmo um filtro ótimo pode ter seu desempenho degradado rapidamente.

Assim, entendemos que os estudos abordados por esse trabalho representam uma contribuição importante para o problema de filtragem de sistemas lineares a tempo discreto com saltos markovianos, fornecendo informações relevantes sobre o comportamento do processo de erro.

Como sugestão de trabalhos futuros, propomos neste mesmo cenário a análise de estabilidade para filtros lineares gerais, sem a hipótese de markovianidade. Também acreditamos que seja importante investigar condições necessárias e suficientes para a estabilidade do filtro, provavelmente usando uma noção do tipo M-estabilizabilidade que generalize M-alcançabilidade. 


\section{Referências Bibliográficas}

[1] S. Aberkane and V. Dragan. H infinity filtering of periodic markovian jump systems: Application to filtering with communication constraints. Automatica, 48:31513156, 2012.

[2] B. D. O. Anderson and J. B. Moore. Optimal Filtering. Prentice-Hall, London, first edition, 1979.

[3] M. Athans, D. Castanon, K. P. Dunn, C. S. Greene, W. H. Lee, N. R. Sandell, and A. S. Willsky. The stochastic control of the F-8C aircraft using a multiple model adaptive control (MMAC) method - Part i :Equilibrium flight. IEEE Transactions on Automatic Control, (22):798-780, 1977.

[4] E. Cinlar. Introduction to Stochastic Processes. Prentice-Hall, 1975.

[5] T. Cormen, C. Leiserson, and C. Stein R. Rivest. Introduction to Algorithms. McGraw-Hill, 2001.

[6] E. F. Costa and A Astolfi. A bound for the error covariance of the recursive Kalman filter with Markov jump parameters. In 47th IEEE Conference on Decision and Control,, pages 37-42, 2009.

[7] E. F. Costa and A. Astolfi. Characterization of exponential divergence of the Kalman filter for time-varying systems. SIAM Journal on control and optimazation, 48(5):2917-2944, 2009.

[8] E. F. Costa and A. Astolfi. A necessary and sufficient condition for exponential diver- 
gence of the recursive Kalman filter for linear time-varying systems. SIAM Journal on Control and Optimization, 48:2917-2944, 2009.

[9] E. F. Costa and A. Astolfi. Stochastic detectability and mean bounded error covariance of the recursive kalman filter with markov jum parameters. Stochastic analysis and Aplications, 28(2):190-201, 2010.

[10] E. F. Costa and J. B. R. do Val. On the detectability and observability of discrete-time Markov jump linear systems. System and Control Letters, 44:135-145, 2001.

[11] E. F. Costa and J. B. R. do Val. On the observability and detectability of continuoustime markov jump linear systems. Society for Industrial and Applied Mathematics, 41:1295-1314, 2002.

[12] E. F. Costa and J. B. R. do Val. Weak detectability and the linear quadratic control problem of discrete-time Markov jump linear systems. International Journal of Control. Special issue on Switched and Polytopic Linear Systems, 75:1282-1292, 2002.

[13] E. F Costa and J. B. R do Val. Weak detectability and the linear quadratic control problem of discrete time Markov jump linear systems. International journal of control. Special issue on Switched and Polytopic Linear Systems, (75):1282-1292, 2002.

[14] E. F. Costa, A. L. P. Manfrim, and J. B. R. Do Val. Weak controllability and weak stabilizability concepts for linear systems with Markov jump parameters. In Proc. ACC'06 American Control Conference, number 75, pages 1282-1292, Minneapolis, USA, 2006.

[15] J. B. R. do Val Costa and J. C. Geromel. Continuous time state-feedback $H_{2}$-control of Markovian jump linear systems via convex analysis. Automatica, 35(5):259-268, 1999.

[16] O. L. V. Costa. Discrete-times coupled Riccati equations for systems with Markovia switching parameters. Journal of Mathematical Analysis and Applications, (149):197-216, 1983.

[17] O. L. V. Costa. Linear minimum mean square error estimation for discrete- time Markovian jump linear systems. IEEE Transactions on Automatic Control, 39(8):1685-1689, 1994. 
[18] O. L. V. Costa. Linear minimum mean square error estimation for discrete-time Markovian jump linear systems. IEEE Transactions on Automatic Control, 39(8):1685-1689, 1994.

[19] O. L. V. Costa. Stability and control for linear systems with jump Markov pertubations. Stochastic Analysis and Applications, 1(13):91-110, 1995.

[20] O. L. V. Costa and G. R. A. M. Benites. Linear minimum mean square filter for discrete-time linear systems with markov jumps and multiplicative noises. Automatica, 47:466-476, 2011.

[21] O. L. V. Costa and M. D. Fragoso. Stability results for discrete-time linear systems with Markovian jumping parameters. Journal of Mathematical Analysis and Applications, 179:154-178, 1993.

[22] O. L. V. Costa and M. D. Fragoso. Discrete-time LQ-optimal control problems for infinite markov jump parameter systems. IEEE Transactions on Automatic Control, 40:2076-2088, 1995.

[23] O. L. V. Costa, M. D. Fragoso, and R. P. Marques. Discrete-Time Markov Jump Linear Systems. Springer-Verlag, United States of America, second edition, 2005.

[24] O. L. V. Costa, M. D. Fragoso, and R. P. Marques. Discrete-Time Markovian Jump Linear Systems. Springer-Verlag, New York, 2005.

[25] O. L. V. Costa, M. D. Fragoso, and M. G. Todorov. Continuous-Time Markov Jump Linear Systems. Springer, Berlin, Heidelberg, 2013.

[26] O. L. V. Costa and S. Guerra. Stationary filter for linear minimum mean square error estimator of discrete-time Markovian jump systems. Automatic Control, IEEE Transactions on, 47(8):1351-1356, 2002.

[27] O. L. V. Costa and S. G. Jimenez. Stationary filter for linear minimum mean square error estimator of discrete-time Markovian jump systems. IEEE Transactions on Automatic Control, 47(8):1351-1356, 2002.

[28] O. L. V. Costa and R. P. Marques. Robust $H_{2}$-control for discrete-time Markovian jump linear systems. International Journal of Control, 73(1):11-21, 2000. 
[29] O. L. V. Costa and A. Oliveira. Optimal meanvariance control for discrete-time linear systems with markovian jumps and multiplicative noises. Automatica, 48:304-315, 2012.

[30] O. L.V. Costa and G. R.A.M. Benites. Linear minimum mean square filter for discrete-time linear systems with Markov jumps and multiplicative noises. Automatica, 47(3):466 - 476, 2011.

[31] J. C. Geronel Deaecto, G. S. and J. Daafouz. Robust $h_{2}$ switched filter design for discrete-time polytopic linear parameter-varying systems. Signal Processing, 2014.

[32] J. B. R do Val and T. Basar. Receding horizon control of jump linear systems and a macroeconomic policy problem. Journal of Economic Dynamics and Control, 23:1099-1131, 1999.

[33] J. B. R. do val and E. F. Costa. Numerical solutions for the linear-quadratic control problem of Markov jump linear systems and a weak detectability concept. IEEE Transactions on Automatic Control, 114(1):69-96, 2002.

[34] V. Dragan, T. Morozan, and A. M. Stoica. Mathematical methods in robust control of discrete-time linear stochastic systems. Springer, 2009.

[35] V. Dragan and A.M. Stoica. Optimal h2 filtering for a class of linear stochastic systems with sampling. Automatica, 48:24942501, 2012.

[36] M. J. F. Gomes e E. F. Costa. Estabilidade do Filtro de Kalman para Sistemas Lineares com Saltos Markovianos. Dissertação de Mestrado - ICMC/USP, 2010.

[37] J. B. R. do Val E. F. Costa and M. D. Fragoso. A new approach to detectability of discrete-time infinite Markov jump linear systems. SIAM Journal on Control and Optimization, Philadelphia, 43(6):2132-2156, 2005.

[38] M. D. Fragoso and O. L. V. Costa. A unified approach for stochastic and mean square stability of continuous-time linear systems with Markovian jumping parameters and additive disturbances. SIAM Journal on Control and Optimization, 44(4):1165-1191, 2005. 
[39] M. J. F. Gomes and E. F. Costa. On the stability of the recursive Kalman filter with Markov jump parameters. In Proc. ACC'010 American Control Conference, pages 4159-4163, 2010.

[40] M. J. F. Gomes and E. F. Costa. Stability of the Kalman Filter for Markov jump linear systems. In Proc. ECMC'011 Electronic Control, Measurement and Signals Conference, pages 7-12, Liberec, Czech Republic, 2011.

[41] A. C. Harvey. Forecasting, Structural Time Series Models and the Kalman Filter. Cambridge University Press, 1979.

[42] P. J. Hespanha. Linear Systems Theory. Princeton University Press, Princeton, New Jersey, first edition, 2009.

[43] Y. Ji and H. J. Chizeck. Jump linear quadratic gaussian control: Steady-state solution and testable conditions. Control Theory and Advanced Technology, 3(6):289-319, 1990.

[44] S. Mastellone, P. Dorato, and C. T. Abdallah. Finite-time stochastic stability of discrete-time nonlinear systems: Analysis and design. 43th Conference on Decision and Control, 3:2572 - 2577, 2004.

[45] A.R.R. Narváez and E. F. Costa. Alcançabilidade e controlabilidade médias para sistemas lineares com saltos markovianos a tempo contínuo. Tese de doutorado ICMC/USP, 2014.

[46] C. Price. An analysis of divergence problem in the Kalman filter. IEEE Transactions on Automatic Control, 13(6):699-702, 1968.

[47] S. Sangsuk-Iam and T. E. Bullock. Analysis of discrete-time Kalman filtering under incorrect noise covariances. IEEE Transactions on Automatic Control, 35(12):1304-1309, 1990.

[48] G.N. Saridis. Intelligent robotic control. IEEE Transactions on Automatic Control, (28):547-557, 1983.

[49] D. D. Sworder and R. O. Rogers. An LQ-solution to a control problem associated with a solar thermal center receiver. IEEE Transactions on Automatic Control, 10(28):971-978, 1983. 
[50] J. L. Willems and F. M. Callier. Divergence of the stationary Kalman filter for correct and for incorrect noise variances. IEEE Transactions on Automatic Control, 1(9):47-54, 1992.

[51] A.H. Zawiski. Stochastic Processes and Filtering Theory. Academic Press, 1970. 


\section{Índice Remissivo}

Cadeia de Markov, 11

Covariância do erro de estimação, 17

Definição

Estabilidade, 4, 52

M-alcançabilidade, 22

Positividade, 4

Filtros homogêneos, 17

Filtros lineares, 16

Filtros lineares markovianos, 18

M-alcançabilidade

Matrizes, 23

Gramiano, 22

Notação de Landau, 10

Positividade

do processo de erro, 46

do segundo momento, 36

Processo de erro, 3

Segundo momento, 15

SLSM, 15

Soluções homogêneas, 18

Suposição 2, 48
Suposição 1,17

Teorema

do posto completo, 26

Estabilidade, 57

Positividade do processo de erro, 44

Valores nominais, 52 